\title{
Mind-body practices for people living with HIV: a systematic scoping review
}

\author{
Maria Pilar Ramirez-Garcia ${ }^{1,2^{*}}$ (D, Marie-Pier Gagnon ${ }^{3,4}$, Sébastien Colson ${ }^{5}$, José Côté ${ }^{1,2}$, Jorge Flores-Aranda ${ }^{6,7}$ and
} Myriam Dupont ${ }^{8}$

\begin{abstract}
Background: Mind-body practices are frequently used by people living with HIV to reduce symptoms and improve wellbeing. These include Tai Chi, Qigong, yoga, meditation, and all types of relaxation. Although there is substantial research on the efficacy of mind-body practices in people living with HIV, there is no summary of the available evidence on these practices. The aim of this scoping review is to map available evidence of mind-body practices in people living with HIV.
\end{abstract}

Methods: The Arksey and O'Malley (Int J Soc Res Methodol 8:19-32, 2005) methodological framework was used. A search of 16 peer-review and grey literature databases, websites, and relevant journals (1983-2015) was conducted. To identify relevant studies, two reviewers independently applied the inclusion criteria to all abstracts or full articles. Inclusion criteria were: participants were people living with HIV; the intervention was any mind-body practice; and the study design was any research study evaluating one or several of these practices. Data extraction and risk of bias assessment were performed by one reviewer and checked by a second, as needed, using the criteria that Cochrane Collaboration recommends for systematic reviews of interventions (Higgins and Green, Cochrane handbook for systematic reviews of intervention. 2011). A tabular and narrative synthesis was carried out for each mind-body practice.

Results: One hundred thirty-six documents drawing on 84 studies met the inclusion criteria. The most widely studied mind-body practice was a combination of least three relaxation techniques $(n=20)$, followed in declining order by meditation ( $n=17)$, progressive muscle relaxation $(n=10)$, yoga $(n=9)$ and hypnosis $(n=8)$. Slightly over half $(47 / 84)$ of studies used a RCT design. The interventions were mainly (46/84) conducted in groups and most (51/84) included daily individual home practice. All but two studies were unblinded to participants.

Conclusion: The amount of available research on mind-body practices varies by practice. Almost half of the studies in this review were at high risk of bias. However, mindfulness, a combination of least three relaxation techniques and cognitive behavioral strategies, and yoga show encouraging results in decreasing physical and psychological symptoms and improving quality of life and health in people living with HIV. More rigorous studies are necessary to confirm the results of Tai Chi, Qigong, and some relaxation techniques.

Keywords: Mind-body practices, HIV, Tai chi, Yoga, Meditation, Relaxation, Progressive muscle relaxation, Hypnosis, Guided imagery, Autogenic training

\footnotetext{
* Correspondence: Pilar.Ramirez.Garcia@umontreal.ca

${ }^{1}$ Faculty of Nursing, Université de Montréal, Montréal, Canada

${ }^{2}$ Research Center of the Centre Hospitalier de l'Université de Montréal,

Montréal, Canada

Full list of author information is available at the end of the article
}

(c) The Author(s). 2019 Open Access This article is distributed under the terms of the Creative Commons Attribution 4.0 International License (http://creativecommons.org/licenses/by/4.0/), which permits unrestricted use, distribution, and reproduction in any medium, provided you give appropriate credit to the original author(s) and the source, provide a link to the Creative Commons license, and indicate if changes were made. The Creative Commons Public Domain Dedication waiver (http://creativecommons.org/publicdomain/zero/1.0/) applies to the data made available in this article, unless otherwise stated. 


\section{Background}

HIV is currently considered a chronic disease and people living with HIV are living longer [1, 2]. A recent study shows life expectancy in people undergoing antiretroviral therapy (ART) has increased by about 10 years. Twenty-year-olds starting ART in 2008-10 in Europe are expected to live to age 68, while their North Americas peers can hope to reach age 65 [3]. Despite the improved effectiveness of antiretroviral therapy, the quality of life of people living with HIV is strongly affected by the presence of one or more physical and psychological symptoms [4-6]. To reduce symptoms and treatment side effects and to improve general wellbeing, 55-60\% of people living with HIV used complementary health approaches (CHA) [7-10]. They reported using CHA to round out conventional HIV care and antiretroviral treatment $[7,10,11]$. The National Center for Complementary and Integrative Health [12] defines CHA "as a group of health care approaches developed outside of mainstream Western or conventional medicine." Most CHA fall into one of two subgroups: natural products or mind-body practices [12]. Some other CHA are traditional healers, Ayurvedic medicine, and traditional Chinese medicine [12].

Mind-body practices are frequently used by people living with HIV [7, 9, 13]. Mind-body practices users believe that the mind is an important components to wellbeing [14] and they use these practices to play an active role in their health management $[2,14]$. Mind-body practices focus on the interactions between the mind, body, and behavior with the aim of affecting physical functioning and promoting health via the mind. Mind-body practices include Tai Chi, Qigong, yoga, meditation, and all types of relaxation (progressive muscle relaxation, hypnosis, guided imagery, breathing exercises, autogenic training, biofeedback and neurofeedback) [15]. Several systematic reviews show promising results of some mind-body practices in people with chronic disease. Two systematic reviews demonstrate that Tai Chi has beneficial effects on psychological wellbeing for various populations $[16,17]$. Other systematic reviews showed that yoga improves exercise capacity and health-related quality of life in individuals with heart disease, stroke, and chronic obstructive pulmonary disease [18], and that relaxation reduces depression as compared to wait-list, no treatment, or minimal treatment in people diagnosed with depression or high-level symptoms of depression [19]. A RCT evaluating a 20-week intervention of supervised yoga practice found that systolic and diastolic blood pressures improved in people living with HIV at risk of developing cardiovascular disease [20]. Also, a pilot RCT found that daily meditation practice increased quality of life in people living with HIV [21].
Although there is substantial research on the effectiveness of mind-body practices on various populations, there is no summary of the available evidence on mind-body practices in people living with HIV. In addition, a preliminary review of published studies evaluating mind-body practices in people living with HIV found that the designs, interventions, and outcomes of these studies were very heterogeneous. Therefore, this review sought to map available evidence of mind-body practices for people living with HIV. Our study objectives were threefold: to provide an overview of the focus, quantity, and characteristics of existing research; to identify the types of available evidence of each mind-body practice in people living with HIV; and to highlight any gap in current knowledge in the goal of guiding future research.

\section{Methods}

A systematic scoping review was carried out. A scoping review tends to address the broader topics when many different study designs might be applied [22]. Scoping reviews "aim to map rapidly the key concepts underpinning a research area and the main sources and types of evidence available, and can be undertaken as standalone projects in their own right, especially where an area is complex or has not been reviewed comprehensively before" [23].

To produce a thorough and systematic overview of existing research, we adopted the Arksey and O'Malley [22] methodological framework for scoping reviews. This review followed the five stages of this framework: 1) identifying the research question; 2) identifying relevant studies; 3) selecting the studies; 4) charting the data; and 5) collating, summarizing, and reporting results. For Arksey and O'Malley [22] study quality assessment is not part of the scoping review process. However, the lack of a process for assessing the quality of the scoping review is problematic, the danger being that the studies' existence, rather than intrinsic quality, underpins conclusions [24]. Therefore, to identify the types of evidence available on mind-body practices in people living with HIV and to highlight any gaps in current research knowledge, we assessed the risks according to the criteria recommended in the Cochrane Handbook for Systematic Reviews of Interventions [25]. Furthermore, to operationalize the stages of selecting studies, charting data and summarizing results, we followed the Cochrane Collaboration's recommendations for systematic reviews [25].

Before starting our scoping review, we wrote a review protocol describing the rationale and planned methods of the review. This protocol is available by contacting the corresponding author. The manuscript respects the Preferred Reporting Items for Systematic Reviews and Meta-Analyses (PRISMA) statement [26] because 
guidelines for reporting scoping review do not exist yet. However, some PRISMA items for systematic reviews are not appropriate for reporting a scoping review, particularly the items on the objectives, research questions and the conduct of a meta-analysis [27].

\section{Identifying the research question}

Our research question asked "What is known from the existing literature about the effectiveness of mind-body practices for people living with HIV?" Based on the National Center for Complementary and Integrative Health [12] definition, mind-body practices are approaches that use the mind to affect physical functioning and promote health. Mind-body practices include Tai Chi and Qigong, yoga, meditation, and all types of relaxation (progressive muscle relaxation, hypnosis, guided imagery, deep-breathing exercises, autogenic training, biofeedback and neurofeedback). Although acupuncture is generally considered among mind-body practices, it was not included in this review because of its place in traditional Chinese medicine [15].

\section{Identifying relevant studies}

To attempt to answer the research question, we identified primary studies and reviews in the literature, by searching electronic databases, websites, reference lists and key journals. A search strategy for electronic databases was developed according to the research question and key concepts. We used the following search terms: HIV OR AIDS AND mind-body therapy OR Alternative Medicine OR meditation OR yoga OR Tai Chi OR Qigong OR relaxation OR relaxation therapy OR biofeedback training OR biofeedback OR neurofeedback OR autogenic training OR deep-breathing OR guided imagery OR imagery OR progressive relaxation therapy OR muscle relaxation OR Jacobson progressive relaxation OR hypnosis OR hypnotherapy. Table 1 shows our Ovid search strategy. This was adapted for other databases, and details of those strategies may be obtained from the authors on request.

A total of 16 peer-review and grey literature databases from 1983 (when HIV was discovered) to October 2015 were searched. The peer-review database searched were: Cochrane Central Register of Controlled Trials (CCTR), Cochrane Database of Systematic Reviews, Cumulative Index to Nursing and Allied Health Literature (CINAHL), Database of Abstracts of Reviews of Effects (DARE), Health Technology Assessment, EMBASE, MEDLINE, PsycINFO and Web of Knowledge. The grey literature databases searched were: Google Scholar, Scirus, Archimède (Université Laval), Papyrus (Université de Montréal), Dissertations \& Theses (ProQuest), the New York Academy of Medicine Grey Literature and OpenGrey. To identify ongoing clinical studies, we searched the following sites:
ClinicalTrials, World Health Organization International Clinical Trials Registry Platform and Canadian trials. Our goal was to conduct a sensitive, rather than a specific, search of the literature. We enlisted the services of a qualified librarian (MD) to conduct the electronic database search. The last search was run on October 31, 2015.

This search was completed by examining the reference lists and citations of all included studies and by hand searching key journals, such as BMC Complementary and Alternative Medicine, Journal of Evidence-Based Integrative Medicine, The Open Complementary Medicine Journal, Evidence-Based Complementary and Alternative Medicine, Complementary Therapies in Medicine, Complementary Therapies in Clinical Practice, Journal of Alternative and Complementary Medicine, Journal of Complementary \& Integrative Medicine and European Journal of Integrative Medicine. We considered publications in any language, as long as there was an English, French, or Spanish abstract.

\section{Selecting the studies}

The search results were merged using reference management software to remove duplicate records of the same report. To identify relevant studies, we examined titles and abstracts using an eligibility checklist of our specific inclusion criteria. These were: participants were people living with HIV, the intervention was any mind-body practice, and the study design was any research study evaluating one or several of these practices. Following Levac, Colquhoun [28], two reviewers (PRG \& SC) independently applied the inclusion criteria to all records. If the eligibility of a study was uncertain from the abstract, the full article was read. Discrepancies were resolved through discussion. All studies that met the inclusion criteria were eligible for the review.

\section{Charting the data}

We developed an electronic data extraction form in Microsoft Excel with the following items: study ID, aims of the study, study design, total study duration, population, number of participants, setting, country, details and type of intervention (total number of intervention groups, specific intervention, length of intervention, number of contacts, provider and method of delivery), outcomes and time points, scales, and authors' conclusions. Two reviewers (PRG \& MPG) extracted data independently from 10 studies to determine if the approach to data extraction was consistent with the research question and purpose. The data were then extracted by one reviewer (PRG) and checked by another (MPG), as required. Discrepancies were resolved through discussion. 
Table 1 Ovid MEDLINE ${ }^{\oplus}$, In-Process \& Other Non-Indexed Citations and OvidMEDLINE(R) 1946 to Present, search strategy

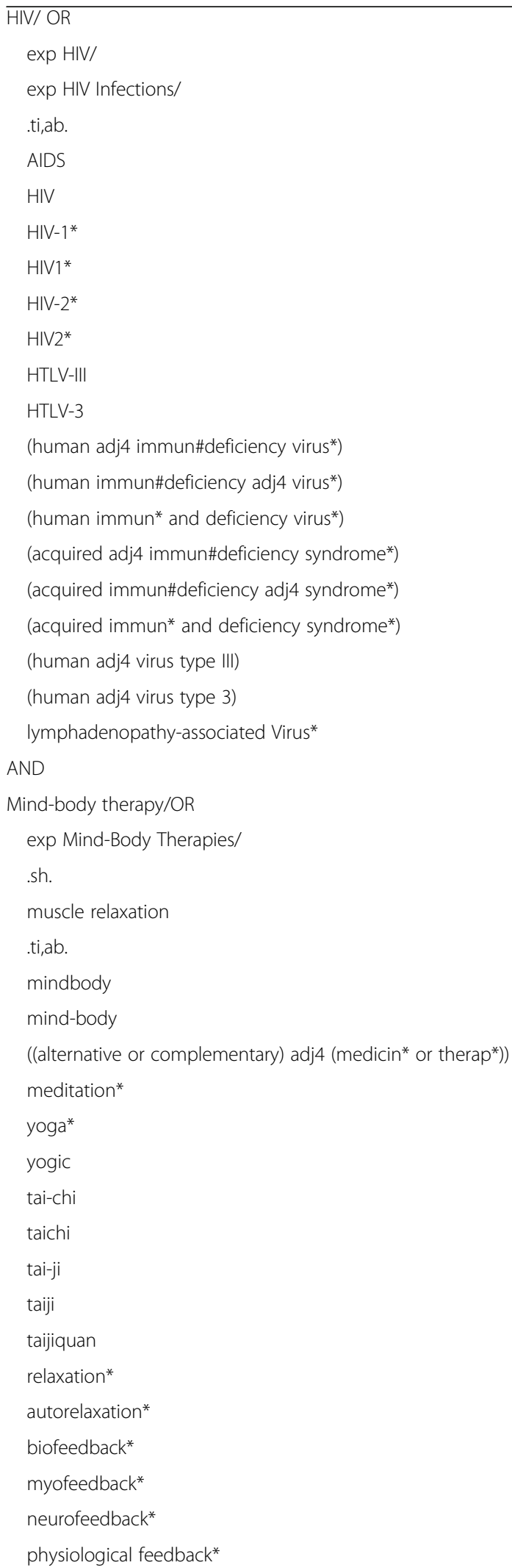

Table 1 Ovid MEDLINE ${ }^{\circledR}$, In-Process \& Other Non-Indexed Citations and OvidMEDLINE(R) 1946 to Present, search strategy (Continued)

autogenic training*
deep-breath*
breathing exercise*
qigong
qi gong
chikung
chi kung
respiratory muscle* training*
imagery
imageries
reverie
visuali\#ation*
hypnos\#s
autohypnos\#s
hypnotherap*
suggestion*
autosuggestion*
mesmerism*

\section{Assessing risk of bias in included studies}

Two reviewers (PRG \& MPG) independently assessed the risk of bias for 10 of the included studies. Risk of bias was then assessed by one reviewer (PRG) and checked by another (MPG), as required. To asses randomized controlled trials (RCTs), we used the criteria recommended in the Cochrane Handbook for Systematic Reviews of Interventions [25] for six domains: selection bias (sequence generation and allocation concealment), performance bias (blinding of participants and health care providers), detection bias (blinding of outcome assessment), attrition bias, selective reporting and other potential sources of bias. Non-randomized controlled trials (NRCT) are defined here as any quantitative study estimating the effectiveness of an intervention that does not use randomization to allocate participants to comparison groups. This includes observational studies, with control before and after the study, interrupted-time-series studies and quasi-randomized studies. To assess the NRCT, we used the criteria recommended in the Cochrane Handbook for Systematic Reviews of Interventions [25] in six domains: selection bias (comparability of groups, confounding and adjustment), performance bias (blinding of participants and health care providers), detection bias (blinding of outcome assessment), attrition bias, selective reporting of outcomes and other potential sources of bias. Qualitative studies were evaluated using the criteria recommended by Spencer et al. [29] in two domains: approach (sampling strategy and sample selection criteria) and credibility (triangulation, respondent 


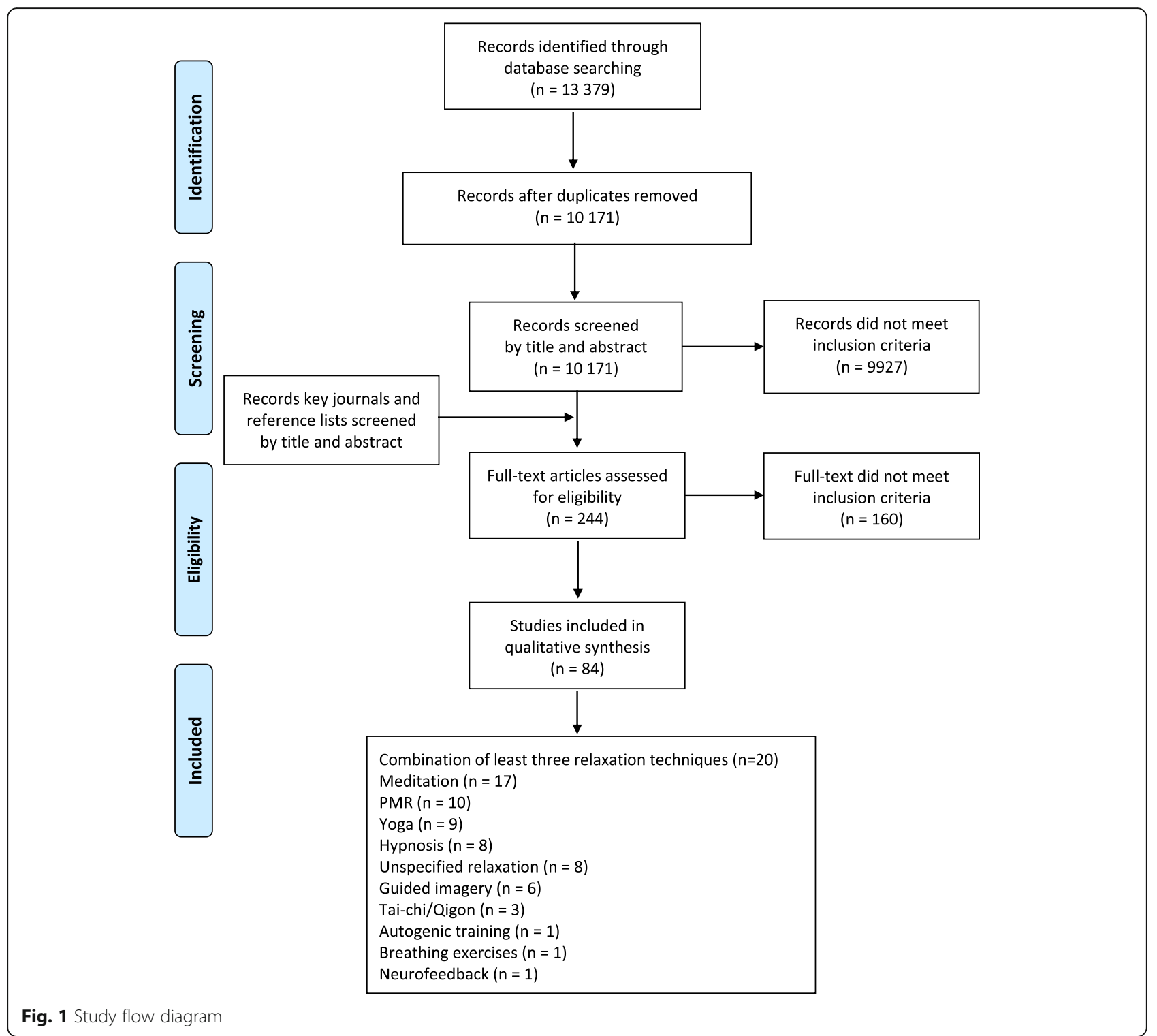

validation, plausibility, presentation of original data and link between data and conclusions).

\section{Collating, summarizing and reporting results}

To answer the research question, we present the details of the included studies in tabular and narrative synthesis form, according to each mind-body practice. The tables include design, sample size, characteristics of intervention, and main results. We classified the studies by period of publication, country of origin, and mind-body practice to show the distribution of studies. For the narrative synthesis, we organized the studies by design, population characteristics, and simple size, and by the nature and characteristics of intervention. In accordance with Higgins and Green [25], the main results from NRCT and RCT are presented separately. The results from the qualitative data are used to explain and complement the results from the quantitative data. To present the evidence available for each mind-body practice and to highlight any gaps in current knowledge with a view to guiding future research, we interpreted the results with a consideration to the studies' bias risk.

\section{Results}

The search strategy identified 13,379 citations (Fig. 1). After removal of 3208 duplicates, 10,171 citations were screened by title and abstract. Of these, 9927 did not meet the criteria for inclusion. Therefore, 244 full text documents retrieved were screened for inclusion in the systematic scoping review. Finally, 136 documents met the inclusion criteria and were included in this systematic scoping review. These were 101 articles, 25 
dissertations, and 10 conference abstracts, and they cover 84 studies (Fig. 1). Multiple documents on the same study were considered for the data extraction and are listed in the tables. The main study is indicated in bold. The most widely studied mind-body practice was a combination of least three relaxation techniques, followed by meditation, progressive muscle relaxation, yoga, hypnosis, guided imagery and Tai Chi/Qigong.

Three quarters of all studies (63/84) were performed in the United States, while seven were conducted in India, and two were carried out in Canada, China, the Netherlands and the United Kingdom. Iran, Japan, New Zealand, South Africa, Thailand and Zambia were the location for one study each. Seven studies were published before 1990. Thereafter, between 12 and 16 studies were published every 5 years, until 2010. Between 2011 and 2015, more than 20 published studies assessed mindbody practices in people living with HIV. Data from these studies show increasing interest in recent years in the HIV population for these types of practices.

We present below the details (design, sample size, intervention and main results by mind-body practice) of the studies included in this review.

\section{Tai Chi and Qigong}

We identified only two published RCTs [30, 31] that assessed the effects of Tai Chi in people living with HIV. We also found one dissertation using a group pretestposttest design to investigate the effects of Qigong on HIV infection [32] (Table 2). One RCT [30] collected qualitative data to explore participants' perceptions of the efficacy of Tai Chi.

The number of participants in two studies [30,32] was close to 30, while McCain et al. (2008) had 200 in their study. Participants were people living with HIV [31,32] or diagnosed with AIDS [30] without significant psychiatric or cognitive impairment [30,31]. The interventions in all three of these studies were conducted in groups. One RCT study compared a short form of Tai Chi of eight movements (TC) with aerobic exercise (EX) and with usual care (CG) [30]. The other RCT compared Tai Chi to an intervention using a relaxation technique and coping strategies for stress management (CBR) with spiritual growth for enhancing exploration of the spiritual self (SP) and with usual care (CG) [31].

Although one RCT [30] was not powered to compare the two intervention groups (13 TC, $13 \mathrm{EX}$, and $12 \mathrm{CG}$ ), both intervention groups showed improvement at the post-intervention checkpoint in physical function, tension/ anxiety, and quality of life compared to the control group. Qualitative data supported the benefits subjects experienced in the experimental groups on physical changes, mood, and social interaction [30]. The other RCT [31] showed a significant decline in usage of emotion-focused coping strategies between the pre-intervention and at the six-month follow-up visits in the TC and CBR groups. Furthermore, all intervention groups (CBR, TC and SP) had increased lymphocyte proliferative function as compared to the control group. The Qigong study [32] found significant differences between pre-intervention and post-intervention in depression and anxiety scores and in T-cell counts. The two RCTs studies were unblinded to participants, but one [31] was blinded to outcome assessors. Two studies [30, 32] presented high risk of attrition (Table 3).

\section{Yoga}

We identified 11 papers, including 8 articles and 3 conference abstracts, that described 9 studies assessing the effects of yoga on people living with HIV [20, 35-42]. Five studies used a RCT design [20,38-41], one had a RCT pilot design [42], and three [35-37] a one-group pretest-posttest design (Table 4). Two studies [36, 38] collected qualitative data to explore the participants' perspective on the yoga's effect on their wellbeing.

Table 2 Tai Chi and Qi gong included studies

\begin{tabular}{|c|c|c|c|c|}
\hline Project identifier & Design & Sample size & Intervention/duration & Outcome/time \\
\hline [32] USA & one group pretest-posttest design & $26 \mathrm{QG}$ & 44 qigong g.s. of $2 \mathrm{~h} / 12$ weeks & $\begin{array}{l}\text { Post-in } \\
\downarrow \text { depression } \\
\downarrow \text { anxiety } \\
\uparrow T \text { cell count }\end{array}$ \\
\hline [30] USA & $\mathrm{RCT}+\mathrm{QA}$ & $\begin{array}{l}13 \mathrm{TC} \\
13 \mathrm{EX} \\
12 \mathrm{CG}\end{array}$ & $\begin{array}{l}16 \text { Tai Chi g.s. } / 8 \text { weeks } \\
16 \text { exercise g.s. } / 8 \text { weeks } \\
\text { usual care }\end{array}$ & $\begin{array}{l}\text { Post-int } \\
\text { TC and EX } \\
\uparrow \text { physical function } \\
\downarrow \text { tension-anxiety } \\
\uparrow \text { QoL }\end{array}$ \\
\hline$[31,33,34]$ USA & RCT & $\begin{array}{l}65 \mathrm{CBR} \\
62 \mathrm{TC} \\
68 \mathrm{SP} \\
57 \mathrm{CG}\end{array}$ & $\begin{array}{l}10 \text { coping and relaxation g.s. } / 10 \text { weeks } \\
10 \text { Tai Chi g.s. } / 10 \text { weeks } \\
10 \text { spiritual growth g.s. } / 10 \text { weeks } \\
\text { usual care }\end{array}$ & $\begin{array}{l}\text { Post-int \& } 6 \text { months } \\
\text { TC and CBR } \\
\downarrow \text { emotion-focused coping } \\
\text { TC, CBR and SP } \\
\uparrow \text { lymphocyte proliferation }\end{array}$ \\
\hline
\end{tabular}

QG qigong, g.s group sessions, Post-int post intervention, $R C T$ randomized control trial, $Q A$ qualitative approach, $T C$ Tai Chi, EX exercise, CG control group, QoL quality of life, CBR cognitive-behavioral relaxation training, SP spiritual growth 
Table 3 Risk of bias of included studies (Quantitative approaches)

\begin{tabular}{|c|c|c|c|c|c|c|}
\hline Project identifier & Selection & Performance & Detection & Attrition & Reporting & Othe \\
\hline \multicolumn{7}{|l|}{ Tai Chi $(n=3)$} \\
\hline \multicolumn{7}{|l|}{$N R C T$} \\
\hline [32] & $H R$ & $H R$ & $H R$ & $H R$ & UR & LR \\
\hline \multicolumn{7}{|l|}{$R C T$} \\
\hline [30] & UR & $H R$ & UR & $H R$ & $L R$ & LR \\
\hline [31] & LR & $H R$ & LR & LR & LR & LR \\
\hline
\end{tabular}

Yoga $(n=9)$

NRCT

\begin{tabular}{|c|c|c|c|c|c|c|}
\hline [35] & HR & $\mathrm{HR}$ & $H R$ & UR & LR & LR \\
\hline [36] & $\mathrm{HR}$ & $\mathrm{HR}$ & $H R$ & $\mathrm{HR}$ & $L R$ & $L R$ \\
\hline [37] & $H R$ & $\mathrm{HR}$ & $\mathrm{HR}$ & UR & LR & LR \\
\hline \multicolumn{7}{|l|}{$C T$} \\
\hline [38] & LR & $\mathrm{HR}$ & UR & $\mathrm{HR}$ & UR & LR \\
\hline [20] & UR & $\mathrm{HR}$ & UR & UR & LR & LR \\
\hline [39] & UR & $\mathrm{HR}$ & UR & $L R$ & LR & $L R$ \\
\hline [40] & UR & $\mathrm{HR}$ & UR & LR & LR & $L R$ \\
\hline [41] & UR & $\mathrm{HR}$ & UR & LR & LR & $L R$ \\
\hline [42] & LR & $\mathrm{HR}$ & UR & $\mathrm{HR}$ & $L R$ & $L R$ \\
\hline
\end{tabular}

Meditation $(n=17)$

NRCT

\begin{tabular}{|c|c|c|c|c|c|c|}
\hline [43] & $\mathrm{HR}$ & $\mathrm{HR}$ & $\mathrm{HR}$ & UR & UR & UR \\
\hline [44] & $\mathrm{HR}$ & $\mathrm{HR}$ & $\mathrm{HR}$ & $\mathrm{HR}$ & LR & LR \\
\hline [45] & $\mathrm{HR}$ & $\mathrm{HR}$ & $\mathrm{HR}$ & UR & LR & LR \\
\hline [46] & $\mathrm{HR}$ & $\mathrm{HR}$ & $\mathrm{HR}$ & UR & LR & LR \\
\hline [47] & $\mathrm{HR}$ & $\mathrm{HR}$ & $\mathrm{HR}$ & LR & LR & LR \\
\hline [48] & $\mathrm{HR}$ & $\mathrm{HR}$ & $\mathrm{HR}$ & UR & UR & UR \\
\hline [49] & $\mathrm{HR}$ & $\mathrm{HR}$ & $\mathrm{HR}$ & $\mathrm{HR}$ & LR & LR \\
\hline [50] & $\mathrm{HR}$ & $\mathrm{HR}$ & $\mathrm{HR}$ & LR & $\mathrm{HR}$ & LR \\
\hline [51] & $\mathrm{HR}$ & $\mathrm{HR}$ & UR & UR & LR & LR \\
\hline \multicolumn{7}{|l|}{$C T$} \\
\hline [52] & UR & $\mathrm{HR}$ & UR & LR & LR & LR \\
\hline [53] & UR & $\mathrm{HR}$ & UR & LR & LR & $L R$ \\
\hline [54] & LR & $\mathrm{HR}$ & UR & LR & LR & $L R$ \\
\hline [55] & UR & $\mathrm{HR}$ & LR & UR & LR & $L R$ \\
\hline [56] & LR & $\mathrm{HR}$ & $\mathrm{HR}$ & $\mathrm{HR}$ & LR & $L R$ \\
\hline [57] & UR & $\mathrm{HR}$ & UR & $L R$ & LR & $L R$ \\
\hline [58] & LR & $\mathrm{HR}$ & UR & $L R$ & LR & LR \\
\hline
\end{tabular}

$\mathrm{PMR}=(9)$

NRCT

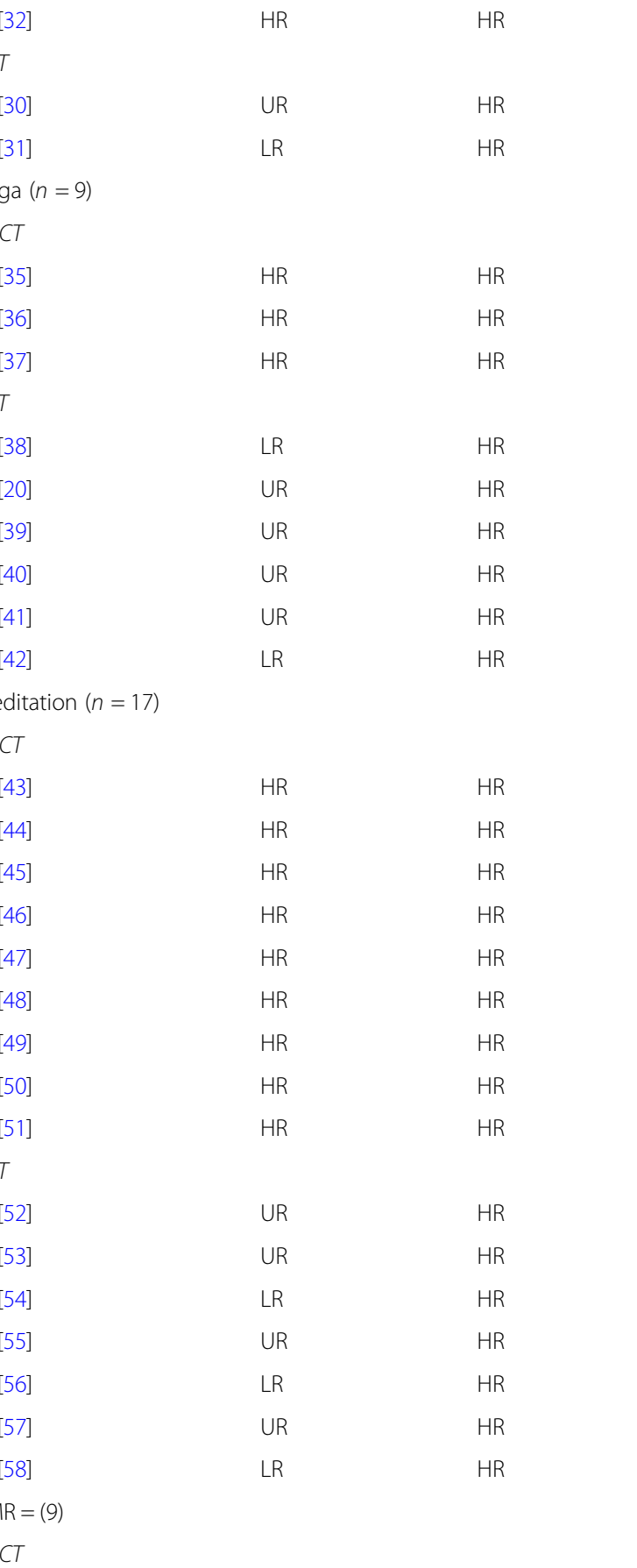

$\mathrm{HR}$

$\mathrm{HR} \quad \mathrm{HR}$

$\mathrm{HR} \quad \mathrm{HR}$

LR

[60]

$H R \quad H R$

$H R$

LR

HR

$H R$

UR

UR

LR

[61]

$H R$

HR

HR

$H R$

UR

UR 
Table 3 Risk of bias of included studies (Quantitative approaches) (Continued)

\begin{tabular}{cllllll}
\hline Project identifier & Selection & Performance & Detection & Attrition & Reporting & Other \\
\hline$[63]$ & UR & HR & UR & UR & LR & LR \\
{$[64]$} & UR & $H R$ & UR & HR & LR \\
{$[65]$} & UR & $H R$ & UR & HR & $L R$ & LR \\
{$[66]$} & HR & HR & UR & UR & HR & UR \\
{$[67]$} & UR & HR & UR & UR & $L R$ & $L R$
\end{tabular}

Hypnosis $(n=8)$

NRCT

\begin{tabular}{|c|c|c|c|c|c|c|}
\hline [68] & $\mathrm{HR}$ & $\mathrm{HR}$ & $\mathrm{HR}$ & $L R$ & $L R$ & $L R$ \\
\hline [69] & $\mathrm{HR}$ & $\mathrm{HR}$ & $\mathrm{HR}$ & $L R$ & LR & LR \\
\hline [70] & $\mathrm{HR}$ & $\mathrm{HR}$ & $\mathrm{HR}$ & $\mathrm{HR}$ & LR & $\mathrm{HR}$ \\
\hline [71] & $\mathrm{HR}$ & $\mathrm{HR}$ & $\mathrm{HR}$ & LR & LR & LR \\
\hline [72] & $\mathrm{HR}$ & $H R$ & UR & UR & LR & LR \\
\hline [73] & LR & $\mathrm{HR}$ & LR & $\mathrm{HR}$ & LR & LR \\
\hline \multicolumn{7}{|l|}{$C T$} \\
\hline [74] & $\mathrm{HR}$ & $\mathrm{HR}$ & LR & $\mathrm{HR}$ & LR & $\mathrm{HR}$ \\
\hline [75] & UR & $\mathrm{HR}$ & UR & UR & UR & UR \\
\hline
\end{tabular}

Guided imagery $(n=6)$

NRCT

\begin{tabular}{|c|c|c|c|c|c|c|}
\hline [76] & $\mathrm{HR}$ & $\mathrm{HR}$ & $\mathrm{HR}$ & $\mathrm{HR}$ & LR & LR \\
\hline [77] & $\mathrm{HR}$ & $\mathrm{HR}$ & $\mathrm{HR}$ & $L R$ & $L R$ & $L R$ \\
\hline [78] & LR & $\mathrm{HR}$ & UR & UR & LR & LR \\
\hline \multicolumn{7}{|l|}{$C T$} \\
\hline [79] & UR & $H R$ & UR & $\mathrm{HR}$ & $L R$ & $L R$ \\
\hline [80] & LR & $\mathrm{HR}$ & UR & LR & $L R$ & LR \\
\hline [81] & LR & $\mathrm{HR}$ & UR & $L R$ & LR & LR \\
\hline
\end{tabular}

Autogenic training $(n=1)$

NRCT

[82]

HR

HR

HR

HR

LR

LR

Breathing exercise $(n=1)$

NRCT

[83]

$H R$

HR

HR

$L R$

LR

$L R$

Neurofeedback $(n=1)$

RCT

[84]

UR

HR

UR

LR

$L R$

LR

Combination of least three relaxation techniques $(n=20)$

NRCT

\begin{tabular}{|c|c|c|c|c|c|c|}
\hline [85] & $\mathrm{HR}$ & $\mathrm{HR}$ & $H R$ & LR & LR & $L R$ \\
\hline [86] & $\mathrm{HR}$ & $H R$ & $\mathrm{HR}$ & $\mathrm{HR}$ & LR & LR \\
\hline [87] & $\mathrm{HR}$ & $\mathrm{HR}$ & $\mathrm{HR}$ & LR & LR & $L R$ \\
\hline [88] & $H R$ & $\mathrm{HR}$ & UR & LR & LR & $L R$ \\
\hline [89] & $\mathrm{HR}$ & $\mathrm{HR}$ & $\mathrm{HR}$ & $\mathrm{HR}$ & LR & LR \\
\hline [90] & LR & UR & UR & $\mathrm{HR}$ & LR & $L R$ \\
\hline & $U \mathrm{~B}$ & $\mathrm{HB}$ & $U \mathrm{~B}$ & 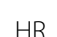 & & \\
\hline
\end{tabular}


Table 3 Risk of bias of included studies (Quantitative approaches) (Continued)

\begin{tabular}{|c|c|c|c|c|c|c|}
\hline Project identifier & Selection & Performance & Detection & Attrition & Reporting & Other \\
\hline [92] & UR & UR & UR & UR & UR & UR \\
\hline [93] & UR & $H R$ & UR & $\mathrm{HR}$ & $L R$ & LR \\
\hline [94] & UR & $H R$ & UR & $L R$ & $L R$ & LR \\
\hline$[95]$ & LR & $\mathrm{HR}$ & UR & $\mathrm{HR}$ & LR & $L R$ \\
\hline$[96]$ & UR & $H R$ & UR & $\mathrm{HR}$ & $L R$ & $L R$ \\
\hline [97] & UR & $H R$ & UR & $L R$ & LR & $L R$ \\
\hline [98] & UR & $\mathrm{HR}$ & UR & $\mathrm{HR}$ & LR & $L R$ \\
\hline [99] & UR & $H R$ & UR & $\mathrm{HR}$ & $L R$ & $L R$ \\
\hline [100] & LR & $\mathrm{HR}$ & LR & $L R$ & LR & $L R$ \\
\hline [101] & $L R$ & $L R$ & UR & $L R$ & $L R$ & $L R$ \\
\hline [102] & UR & $\mathrm{HR}$ & UR & $\mathrm{HR}$ & LR & $L R$ \\
\hline [103] & UR & $H R$ & UR & $\mathrm{HR}$ & $L R$ & $L R$ \\
\hline [104] & LR & $L R$ & UR & $L R$ & LR & $L R$ \\
\hline \multicolumn{7}{|c|}{ Unspecified relaxation $(n=8)$} \\
\hline \multicolumn{7}{|l|}{ NRCT } \\
\hline$[105]$ & $\mathrm{HR}$ & $\mathrm{HR}$ & $\mathrm{HR}$ & $\mathrm{HR}$ & LR & $L R$ \\
\hline$[106]$ & UR & UR & UR & UR & $L R$ & $L R$ \\
\hline \multicolumn{7}{|l|}{$R C T$} \\
\hline [107] & UR & $H R$ & UR & UR & UR & $L R$ \\
\hline [108] & UR & $H R$ & UR & $H R$ & $L R$ & $L R$ \\
\hline [109] & UR & $H R$ & UR & UR & $L R$ & $L R$ \\
\hline [110] & UR & $\mathrm{HR}$ & UR & $H R$ & $L R$ & $L R$ \\
\hline [111] & UR & $H R$ & UR & LR & $L R$ & $L R$ \\
\hline [112] & $H R$ & $H R$ & UR & $H R$ & $L R$ & $L R$ \\
\hline
\end{tabular}

Three studies had fewer than 15 participants per group [36, 40, 42]. The number of participants per group in the other studies ranged from $20[20,38]$ to 96 [37]. Most were conducted on samples of people living with HIV [35] in stable condition and without significant psychiatric or cognitive impairment [20, $38]$ or cardiac problems [20, 41]. One study was conducted with participants with pain or anxiety [36] and another [42] with people living with HIV who used crack cocaine. One study was conducted with adolescents, aged between 11 and 16 years, who were aware of their HIV status and had been receiving antiretroviral treatment for at least 1 year [40]. Four out of five RCTs compared yoga intervention to usual care. One RCT [40] compared a yoga and peer-support intervention to peer-support intervention only and to usual care. Six studies [35, 36, 38, 40-42] evaluated yoga in groups. The intensity and duration of interventions varied between 10 and 90 sessions within a 4-26-week period; the most frequent duration was, however, between 8 and 12 weeks.
One-group studies showed post-intervention effects on pain, anxiety [36], and CD4 counts [37]. RCTs showed that yoga could decrease psychological distress $[40,42]$, and increase quality of life $[38,41]$ and CD4 counts [40] immediately after the intervention. Qualitative data suggest that participants perceived yoga as a way to gain awareness and constructively manage the physical and emotional challenges related to their HIV $[36,38]$. One RCT [20] showed a decrease in trans-fat consumption and in participants' blood pressure after the group's yoga intervention, as compared to the control group. Only one pilot RCT [42] showed that the effects of yoga on psychological distress were maintained 2 months after the intervention. All RCTs were unblinded to participants. They did not give information on blinding of outcome assessors. Three studies [36, 38, 42] presented high risk of attrition (Table 3).

\section{Meditation}

We identified 23 papers (17 articles, 5 dissertations and 1 conference abstract) reporting on 17 studies that 
Table 4 Yoga included studies

\begin{tabular}{|c|c|c|c|c|}
\hline Project identifier & Design & $\begin{array}{l}\text { Sample } \\
\text { size }\end{array}$ & Intervention/duration & Outcome/time \\
\hline [35] USA & $\begin{array}{l}\text { one group pretest-posttest } \\
\text { design }\end{array}$ & 48 BMSG & 10 yoga, nutrition, hardiness, alternatives therapies g.s. /10 weeks & Post-int \\
\hline [36] USA & $\begin{array}{l}\text { one group pretest-posttest } \\
\text { design + QA }\end{array}$ & $4 Y G$ & 16 lyengar yoga g.s. /8 weeks & $\begin{array}{l}\text { Post-int } \\
\downarrow \text { pain } \\
\downarrow \text { anxiety }\end{array}$ \\
\hline [37] India & $\begin{array}{l}\text { one group pretest-posttest } \\
\text { design }\end{array}$ & 96 YGNA & $\begin{array}{l}\text { Twice daily yoga, hydrotherapy, diet therapy and sun bath } / 1 \text { to } \\
180 \text { days - (average- } 4 \text { weeks) }\end{array}$ & $\begin{array}{l}\text { Post-int } \\
\uparrow \text { CD4 counts }\end{array}$ \\
\hline \multirow[t]{2}{*}{ [38] Canada } & \multirow[t]{2}{*}{$\mathrm{RCT}+\mathrm{QA}$} & $20 Y G$ & 27 yogic breathing and meditation g.s. $/ 14$ weeks & \multirow{2}{*}{$\begin{array}{l}\text { Post-int } \\
\uparrow \text { well-being }\end{array}$} \\
\hline & & $27 C G$ & usual care & \\
\hline \multirow[t]{2}{*}[20,113]{ USA } & \multirow[t]{2}{*}{$\mathrm{RCT}$} & 29 YG & $\begin{array}{l}18 \text { Ashtanga Vinyasa yoga i.s. + } 22 \text { Ashtanga Vinyasa yoga g.s. } \\
\text { /20 weeks }\end{array}$ & \multirow{2}{*}{$\begin{array}{l}\text { Post-int } \\
\downarrow \text { trans fat } \\
\text { intake } \\
\downarrow \text { blood } \\
\text { pressure }\end{array}$} \\
\hline & & $21 C G$ & usual care & \\
\hline \multirow{2}{*}{$\begin{array}{l}\text { [39] conference } \\
\text { abstract India }\end{array}$} & \multirow[t]{2}{*}{$\mathrm{RCT}$} & YG 35 & 90 yoga s. $/ 12$ weeks & \multirow{2}{*}{$\begin{array}{l}\text { Post-int } \\
\downarrow \text { distress }\end{array}$} \\
\hline & & CG 35 & usual care & \\
\hline \multirow[t]{4}{*}[40,114]{ Zambia } & \multirow[t]{4}{*}{$\mathrm{RCT}$} & adolescents & & \multirow{4}{*}{$\begin{array}{l}\text { Post-int } \\
\downarrow \text { emotional } \\
\text { symptom } \\
\uparrow \text { CD4 counts }\end{array}$} \\
\hline & & 12 YGPS & 20 yoga g.s. and group discussion $/ 10$ weeks & \\
\hline & & 11 PS & 20 group discussion $/ 10$ weeks & \\
\hline & & $11 C G$ & usual care & \\
\hline \multirow[t]{2}{*}{ [41] India } & \multirow[t]{2}{*}{$\mathrm{RCT}$} & 31 YG & 12 sudarshan kriya yoga g.s. of $2 \mathrm{~h} / 12$ weeks & \multirow{2}{*}{$\begin{array}{l}\text { Post-int } \\
\uparrow \text { QoL }\end{array}$} \\
\hline & & 30 CG & usual care & \\
\hline \multirow[t]{2}{*}{ [42] USA } & \multirow[t]{2}{*}{ pilot RCT } & $12 Y G$ & 16 yoga g.s. $/ 8$ weeks & \multirow{2}{*}{$\begin{array}{l}\text { Post-int \& } 4 \\
\text { months } \\
\text { Feasible and } \\
\text { acceptable } \\
\downarrow \text { distress } \\
\downarrow \text { stress }\end{array}$} \\
\hline & & $12 C G$ & usual care & \\
\hline
\end{tabular}

BMSG behavioral medicine support group, g.s group sessions, Post-int: post-intervention, $Q A$ qualitative approach, $Y G$ yoga, $R C T$ randomized control trial, $C G$ control group, i.s individual sessions, YGPS yoga and peer support, PS pair support, YGNA yoga and naturopathy interventions, QoL quality of life

assessed any form of meditation in people living with HIV. Seven studies used a RCT design [52-58], one had a pilot RCT design [21], six used one-group pretest-posttest design [43-48], two had a non-equivalent untreated control group with pretest and posttest design $[49,51]$ while one action research design was built with mixed methods [50]. Five studies collected qualitative data to explore the perceived benefit from meditation to the participants (Table 5).

Most studies were conducted on general samples of people living with HIV [21, 46, 47, 49, 50, 52, 54, 56] with moderate distress [44, 55] or ARV side effects [57]. One study was conducted on drug users living with HIV who were enrolled in an inner-city methadone maintenance program; [51] another on gay men living with HIV [58], and a third on near end-of-life AIDS patients [53]. The participants of one study were adolescents (aged 13-21), a third of whom was living with HIV [45]. One study included people who had never tested, who were HIV-, HIV+ or who were living with AIDS [43]. Most studies excluded people with cognitive impairment, psychosis, or substance use. The number of participants ranged from 5 [44] to 245 [56]. Seven studies had fewer than 15 participants per group [21, $44,46-48,52,53]$. The number of participants per group in other studies ranged from 15 [55] to 125 [56]. Six studies assessed the effect of a mindfulness-based stress reduction (MBSR) intervention [45, 49, 55-58]. The MBSR group intervention consisted of eight weekly sessions of 2-3-h each, a daylong retreat, and daily home practice. MBSR meditation practices include body-scan meditation, yoga postures practiced with mindful awareness of the body, and sitting meditation with mindfulness of breath, thoughts, and emotions. The other forms of meditation assessed were mantra repetition [43, 54]. Metta meditation [53], transcendental meditation [21] and any form of mindfulness training combined with other cognitive, emotional, or spiritual techniques [44, 46-48, 50, 51]. A study [52] assessed an intervention with two components: a cognitive-behavioral stress training and Benson relaxation response. The duration of these interventions varied 
Table 5 Meditation included studies

\begin{tabular}{|c|c|c|c|c|}
\hline $\begin{array}{l}\text { Project } \\
\text { identifier }\end{array}$ & Design & $\begin{array}{l}\text { Sample } \\
\text { size }\end{array}$ & Intervention/duration & Outcome/time \\
\hline [43] USA & $\begin{array}{l}\text { one group pretest-posttest } \\
\text { design }\end{array}$ & $175 \mathrm{MD}$ & 10 meditation i.s. + mantra's repetition daily /10 weeks & $\begin{array}{l}\text { Post-int } \\
\downarrow \text { distress }\end{array}$ \\
\hline [44] USA & $\begin{array}{l}\text { one group pretest-posttest } \\
\text { pilot design }\end{array}$ & $5 \mathrm{MBCT}$ & $\begin{array}{l}8 \text { mindfulness-based stress reduction and cognitive therapy g.s and } 1 \\
\text { mindfulness-based stress reduction and cognitive therapy i.s. + daily } \\
\text { practice / } 8 \text { weeks }\end{array}$ & $\begin{array}{l}\text { Post-int \& } 4 \\
\text { month } \\
\downarrow \text { depressive } \\
\text { symptoms } \\
\uparrow \text { dispositional } \\
\text { mindfulness } \\
\uparrow \text { QoL }\end{array}$ \\
\hline $\begin{array}{l}{[45,115,116]} \\
\text { USA }\end{array}$ & $\begin{array}{l}\text { one group pretest-posttest } \\
\text { pilot design + QA }\end{array}$ & $\begin{array}{l}\text { adolescents } \\
59(11 \mathrm{HIV}+) \\
\text { MBSR }\end{array}$ & $\begin{array}{l}9 \text { body scan, mindfulness meditation, yoga g.s of } 2 \mathrm{~h}+\text { daily practice } \\
/ 8 \text { weeks }\end{array}$ & $\begin{array}{l}\text { Post-int } \\
\text { Feasible and } \\
\text { acceptable } \\
\downarrow \text { symptoms } \\
\text { (hostility and } \\
\text { discomfort) }\end{array}$ \\
\hline [46] USA & $\begin{array}{l}\text { one group pretest-posttest } \\
\text { pilot design + QA }\end{array}$ & $11 \mathrm{MCPA}$ & $\begin{array}{l}5 \text { noticing positive events, capitalizing, gratitude, mindfulness, positive } \\
\text { reappraisal, personal strengths, attainable goals, kindness, i.s. + daily } \\
\text { home practice } / 6 \text { weeks }\end{array}$ & $\begin{array}{l}\text { Post-int } \\
\text { Feasible and } \\
\text { acceptable } \\
\uparrow \text { positive affect } \\
\downarrow \text { negative affect }\end{array}$ \\
\hline [47] USA & $\begin{array}{l}\text { one group pretest-posttest } \\
\text { pilot design + QA }\end{array}$ & 9 MABT & $\begin{array}{l}8 \text { massage, interceptive training, mindful body awareness i.s. of } 1 \\
\text { h30 + home practice } / 8 \text { weeks }\end{array}$ & $\begin{array}{l}\text { Post-int } \\
\text { Feasible and } \\
\text { acceptable }\end{array}$ \\
\hline $\begin{array}{l}{[48]} \\
\text { Conference } \\
\text { abstract UK }\end{array}$ & $\begin{array}{l}\text { one group pretest-posttest } \\
\text { pilot design + QA }\end{array}$ & 12 CFT & $\begin{array}{l}8 \text { attention, emotional regulation, mindfulness practice g.s. of } 2 \mathrm{~h} / 8 \\
\text { weeks }\end{array}$ & $\begin{array}{l}\text { Post-int } \\
\text { Feasible and } \\
\text { acceptable }\end{array}$ \\
\hline \multirow[t]{2}{*}{ [49] USA } & \multirow[t]{2}{*}{$\begin{array}{l}\text { untreated control group with } \\
\text { pretest and posttest design }\end{array}$} & $24 \mathrm{MBSR}$ & $\begin{array}{l}8 \text { body scan, mindfulness meditation, hatha yoga g.s. of } 2 \text { h } 30+\text { daily } \\
\text { home practice } \\
\text { / } 8 \text { weeks }\end{array}$ & \multirow{2}{*}{$\begin{array}{l}\text { Post-int \& } 5 \\
\text { months } \\
\downarrow \text { mood } \\
\text { disturbance } \\
\downarrow \text { stress } \\
\uparrow \text { NK cell } \\
\text { number and } \\
\text { activity }\end{array}$} \\
\hline & & $10 \mathrm{CG}$ & usual care & \\
\hline [50] Thailand & $\begin{array}{l}\text { action research: mixed } \\
\text { method }\end{array}$ & 16 PSM & $\begin{array}{l}8 \text { mindfulness meditation, emotional reflective techniques s. + daily } \\
\text { home practice / } 8 \text { weeks }\end{array}$ & $\begin{array}{l}\text { Post-int } \\
\downarrow \text { suffering }\end{array}$ \\
\hline \multirow[t]{2}{*}[51,117]{ USA } & \multirow{2}{*}{$\begin{array}{l}\text { untreated control group with } \\
\text { pretest and posttest design }+ \\
\text { QA }\end{array}$} & $2135+$ & $\begin{array}{l}12 \text { cognitive-behavioral spirituality focused psychotherapy, } \\
\text { mindfulness training i.s. + daily mindfulness home practice } / 12 \text { weeks }\end{array}$ & \multirow{5}{*}{$\begin{array}{l}\text { Post-int } \\
\downarrow \text { impulsivity } \\
\uparrow \text { spirituality }\end{array}$} \\
\hline & & 17 CG & usual care & \\
\hline \multirow[t]{3}{*}{ [52] USA } & \multirow[t]{3}{*}{$\mathrm{RCT}$} & $10 \mathrm{ST}$ & 4 support group g.s. of 1 h30 /4 weeks & \\
\hline & & 10 CBST & $\begin{array}{l}4 \text { Benson's relaxation exercises, cognitive-behavioral stress training g.s. } \\
\text { of } 1 \text { h30 + twice daily home practice/ } 4 \text { weeks }\end{array}$ & \\
\hline & & $10 \mathrm{WL}$ & wait-list & \\
\hline \multirow[t]{4}{*}{ [53] USA } & \multirow[t]{4}{*}{$\mathrm{RCT}$} & $13 \mathrm{MT}$ & 1 meditation g.s. of 90 min + daily meditation home practice $/ 4$ weeks & \multirow{4}{*}{$\begin{array}{l}\text { Post-int \& } 2 \\
\text { months } \\
\text { To MT + MSSG: } \\
\uparrow \text { QoL }\end{array}$} \\
\hline & & 13 MSSG & 20 massage i.s. of $30 \mathrm{~min} / 4$ weeks & \\
\hline & & $\begin{array}{l}16 \mathrm{MT}+ \\
\mathrm{MSSG}\end{array}$ & $\begin{array}{l}1 \text { meditation g.s of } 90 \mathrm{~min}+\text { daily meditation home practice }+ \text { and } 20 \\
\text { massage i.s of } 30 \mathrm{~min} / 4 \text { weeks }\end{array}$ & \\
\hline & & 16 Control & usual care & \\
\hline \multirow[t]{2}{*}[54,118]{ USA } & \multirow[t]{2}{*}{$\mathrm{RCT}$} & $46 \mathrm{MT}$ & $\begin{array}{l}6 \text { mantra's repetition g.s. of } 90 \text { min and group discussion + daily } \\
\text { practice } / 10 \text { weeks }\end{array}$ & \multirow{2}{*}{$\begin{array}{l}\text { Post-int \& } 22 \\
\text { weeks } \\
\downarrow \text { anger } \\
\uparrow \text { spiritual } \\
\text { wellbeing }\end{array}$} \\
\hline & & $47 \mathrm{ACG}$ & $\begin{array}{l}6 \text { videotapes on HIV-topics g.s. of } 90 \text { min and group discussion /10 } \\
\text { weeks }\end{array}$ & \\
\hline \multirow[t]{2}{*}[55,119]{ USA } & \multirow[t]{2}{*}{$\mathrm{RCT}$} & $33 \mathrm{MBSR}$ & $\begin{array}{l}8 \text { body scan, mindfulness meditation, yoga g.s. of } 2 \mathrm{~h}+\text { a day long } \\
\text { retreat }(6 \mathrm{~h})+\text { daily practice } / 10 \text { weeks }\end{array}$ & \multirow[t]{2}{*}{$\begin{array}{l}\text { Post-int } \\
=\text { CD4 counts }\end{array}$} \\
\hline & & 15 CG & 1 day MBSR information & \\
\hline
\end{tabular}


Table 5 Meditation included studies (Continued)

\begin{tabular}{|c|c|c|c|c|}
\hline $\begin{array}{l}\text { Project } \\
\text { identifier }\end{array}$ & Design & $\begin{array}{l}\text { Sample } \\
\text { size }\end{array}$ & Intervention/duration & Outcome/time \\
\hline \multirow[t]{2}{*}[56,120]{$\operatorname{Iran}$} & \multirow[t]{2}{*}{$\mathrm{RCT}$} & $120 \mathrm{MBSR}$ & $\begin{array}{l}8 \text { body scan, mindfulness meditation, yoga g.s. of } 2 h+1 \text { day of } \\
\text { retreat }(6 h)+\text { daily practice } / 8 \text { weeks }\end{array}$ & \multirow{2}{*}{$\begin{array}{l}\text { Post-int \& } 12 \\
\text { months } \\
\downarrow \text { physical } \\
\text { symptoms }\end{array}$} \\
\hline & & $125 \mathrm{ESC}$ & 2 HIV education g.s of $2 \mathrm{~h}$ & \\
\hline \multirow[t]{2}{*}{ [57] USA } & \multirow[t]{2}{*}{$\mathrm{RCT}$} & $40 \mathrm{MBSR}$ & $\begin{array}{l}8 \text { body scan, mindfulness meditation, yoga g.s. of } 2 \text { h30 }+1 \text { day of } \\
\text { retreat }(6 \mathrm{~h})+\text { daily practice } / 8 \text { weeks }\end{array}$ & \multirow{2}{*}{$\begin{array}{l}\text { Post-int } \\
\downarrow \text { ARV side } \\
\text { effects }\end{array}$} \\
\hline & & 36 CG & wait-list & \\
\hline \multirow[t]{2}{*}{ [58] Canada } & \multirow[t]{2}{*}{$\mathrm{RCT}$} & $78 \mathrm{MBSR}$ & $\begin{array}{l}8 \text { body scan, mindfulness meditation, yoga g.s. of } 3 h+1 \text { day of } \\
\text { retreat }(6 h)+\text { daily practice } / 8 \text { weeks }\end{array}$ & \multirow{2}{*}{$\begin{array}{l}\text { Post-int \& } 6 \\
\text { months } \\
\downarrow \text { avoidance } \\
\uparrow \text { positive affect } \\
\uparrow \text { mindfulness }\end{array}$} \\
\hline & & 39 CG & usual care & \\
\hline \multirow[t]{2}{*}{ [21] USA } & \multirow[t]{2}{*}{ pilot RCT } & $11 \mathrm{TM}$ & 14 transcendental meditation s. + daily home practice / 24 weeks & \multirow{2}{*}{$\begin{array}{l}\text { Post-int } \\
\text { Feasible and } \\
\text { acceptable } \\
\uparrow \text { HRQoL }\end{array}$} \\
\hline & & $11 \mathrm{HE}$ & 14 healthy eating education $\mathrm{s}$. & \\
\hline
\end{tabular}

$M D$ meditation, i.s. individual sessions, Post-int post intervention, $M B C T$ mindfulness-based cognitive therapy, g.s group sessions, QoL quality of life, $Q A$ qualitative approach, MBSR mindfulness-based stress reduction program, $R C T$ randomized controlled trial, $Q E$ qualitative evaluation, $M C P A$ multiple-component positive affect, MABT mindful awareness in body-oriented therapy, CFT compassion-focused therapy, 3-S+ spiritual self-schema therapy, PSM palliative-suffering model, TM transcendental meditation, $H E$ healthy eating education, $H R Q L L$ health related quality of life, ST support training, SIT stress inoculation training, WL wait list, MT mantra, MSSG massage, MT + MSSG mantra + massage, ACG attention control group, CG control group, ESC education and support condition

between $4[52,53]$ and 24 weeks [21], but the most frequent duration was between 8 and 12 weeks [44, 47, 50, 51, 54]. All the interventions included daily home practice.

One group pretest-posttest design found that mantra repetition reduced distress in people who had never tested for HIV, HIV- people and HIV+ people [43]. Two NRCTs studies found that MBSR reduced hostility, discomfort symptoms [45], mood disturbance and stress [49]. Qualitative data suggested that MBSR helped participants feel calmer, happier and more able to manage their anger and conflicts [45]. Other very small NCRTs $(n=5)$ found that mindfulness training combined with other cognitive, emotional, or spiritual techniques reduced depression symptoms [44], negative affects [46], and impulsivity [51], and it increase quality of life [44]. Participants in an intervention using any form of mindfulness training and massage explained that they had learned self-care skills based on self-awareness, and that the intervention had had a positive effect on their symptom management and on facing up to the challenges of living with HIV [47]. Similarly, participants in an intervention combining mindfulness meditation with emotional reflective techniques stated that the intervention helped them understand and accept the nature of life, manage their behaviors, and initiate self-healing to alleviate their suffering [50].

Three RCTs studies found that MBSR reduced physical symptoms [56] and ARV side effects [57], and increased positive affect at post-intervention and at 6 months [58]. One RCT founds that mantra repetition reduce distress and increase spiritual wellbeing immediately after the intervention and after 3 months [54]. A small RCT ( $n=$ 42) [53] found that meditation combined with massage was more effective in increasing the quality of life of people living with HIV approaching end of life than meditation or massage alone. Finally, a pilot RCT found that daily practice of transcendental meditation could be effective to increase quality of life in people living with HIV [21]. All studies were unblinded to participants and only two studies $[21,55]$ were blinded to outcome assessors. Three studies $[44,49,56]$ presented high risk of attrition (Table 3).

\section{Relaxation}

A total of 97 documents were reviewed. These consisted of 73 articles, 19 dissertations, and 5 conference abstracts drawing on 55 studies that assessed one or more relaxation techniques in people living with HIV. Ten of these studies assessed progressive muscle relaxation, eight examined hypnosis, and six looked at guided imagery. Only one study was found for breathing exercises, autogenic training, and neurofeedback techniques. Additionally, 20 studies assessed a combination of at least 3 relaxation techniques with or without other components. Finally, eight studies assessed a relaxation technique combined with cognitive behavioral therapy, without however specifying the relaxation technique used. The following sections present the main findings of these studies.

\section{Progressive muscle relaxation}

We identified 16 papers, including 12 articles, 3 dissertations and 1 conference abstract, discussing progressive 
muscle relaxation effects in people living with HIV, as presented in 10 different studies [59-67, 121-127]. Five studies used a RCT design [63-67], three used one-group pretest-posttest design [59-61], and one had a phenomenological design [121]. One study [62] began by randomizing participants into three groups. However, since almost half of the subjects assigned to group intervention refused to participate, they were reassigned to the comparison group (Table 6).

Six studies had fewer than 15 participants per group $[59,60,62,65-67]$, while 4 had between 15 and 30 participants in each group [61, 63, 64, 121]. Four studies recruited people living with HIV who showed a stable condition $[61,66,67,121]$ and two studies recruited people with symptomatic HIV infection [59, 62]. The population of three studies consisted of peopling living with HIV who presented problems related to anxiety or depression $[59,62]$. One study enrolled healthy homosexual men who were unaware of their HIV-1 antibody status [63]. In general, participants with any history of drug or alcohol abuse $[63,65]$ or with any current and major psychiatric disorder $[59,60,62,65]$ were excluded. Four studies offered group interventions [62-65], while three had individual interventions, and $[59,60,67]$ one offered either individual or group intervention [121]. Two studies did not specify their offering $[61,66]$. The number of meetings varied between a one-time 30-min meeting [121] and nearly twenty 20-90-min meetings $[59,63,67]$ over a period of 8 [64] to 20 weeks [59]. Six studies combined progressive muscle relaxation with different cognitive behavioral strategies $[59,60,62-65]$ and offered daily relaxation practice at home [62-65]. Another study combined progressive muscle relaxation with autogenic training [66], while another combined this type of relaxation with guided imagery [121]. Two other studies assessed progressive muscle relaxation as the only intervention applied $[61,67]$.

Combining progressive muscle relaxation with cognitive behavioral strategies, three very small studies with a one-group pretest-posttest design [59, 60, 62] suggest their interventions could reduce physical symptoms, as well as anxiety and depression, and increase patient compliance and quality of life. Participants from a phenomenological study reported felling refreshed and peaceful after 30-min progressive muscle relaxation and guided imagery practice. They also noted release of their physical manifestations of worry, frustration, anxiety and stress [121]. Furthermore, Bommareddi and Valsaraj [61] showed in India that just a 10-week practice of progressive muscle relaxation reduced anxiety and depression in people living with HIV.

Antoni and his colleagues [63] found that an intervention using progressive muscle relaxation and cognitive behavioral strategies reduces the distress and immune system deterioration in gay men associated with notification of the serological status. Moreover, the post-notification distress and immunologic values were strongly associated with self-reports of daily relaxation practice at home. Two RCTs combining progressive muscle relaxation with cognitive behavioral strategies $[64,65]$ showed the beneficial effects of these interventions on symptoms of depression in people living with HIV. A small three-arm RCT [66] compared an intervention combining progressive muscle relaxation and autogenic training (PMR + AT) to psychotherapy (PSY) and to a wait list group (WL) for 12 weeks. The results indicated the effectiveness of relaxation techniques on anxiety, depression, confusion and fatigue, but no significant differences in the effectiveness of relaxation techniques compared to psychotherapy. Finally, comparing massage to progressive muscle relaxation over a 12 -week period in a group of adolescents living with HIV $(n=24)$, a small RCT [67] found beneficial results on depression and on immune system function among the adolescents receiving massage but not those in the relaxation group. All RCTs studies were unblinded to participants and they did not give information on blinding of outcome assessors. Four studies [59, 62, 64, 65] were at high risk of attrition

Antoni and his colleagues [63] found that an intervention using progressive muscle relaxation and $\operatorname{cog}$ nitive behavioral strategies reduces the distress and immune system deterioration in gay men associated with notification of the serological status. Moreover, the post-notification distress and immunologic values were strongly associated with self-reports of daily relaxation practice at home. Two RCTs combining progressive muscle relaxation with cognitive behavioral strategies [64, 65] showed the beneficial effects of these interventions on symptoms of depression in people living with HIV. A small three-arm RCT [66] compared an intervention combining progressive muscle relaxation and autogenic training (PMR $+\mathrm{AT})$ to psychotherapy (PSY) and to a wait list group (WL) for 12 weeks. The results indicated the effectiveness of relaxation techniques on anxiety, depression, confusion and fatigue, but no significant differences in the effectiveness of relaxation techniques compared to psychotherapy. Finally, comparing massage to progressive muscle relaxation over a 12 -week period in a group of adolescents living with HIV $(n=24)$, a small RCT [67] found beneficial results on depression and on immune system function among the adolescents receiving massage but not those in the relaxation group. All RCTs studies were unblinded to participants and they did not give information on blinding of outcome assessors. Four studies [59, 62, 64, 65] were at high risk of attrition (Table 3). 


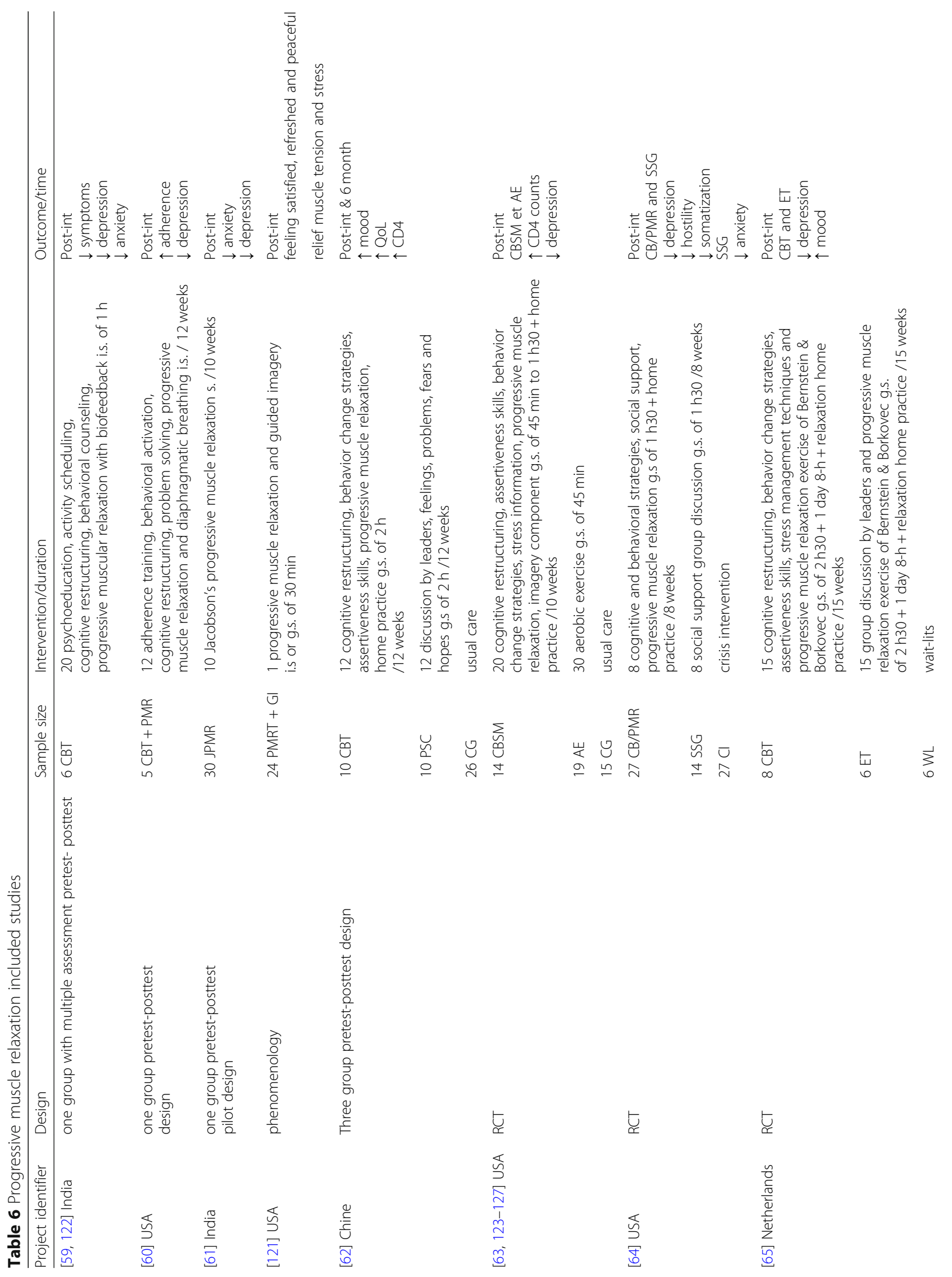




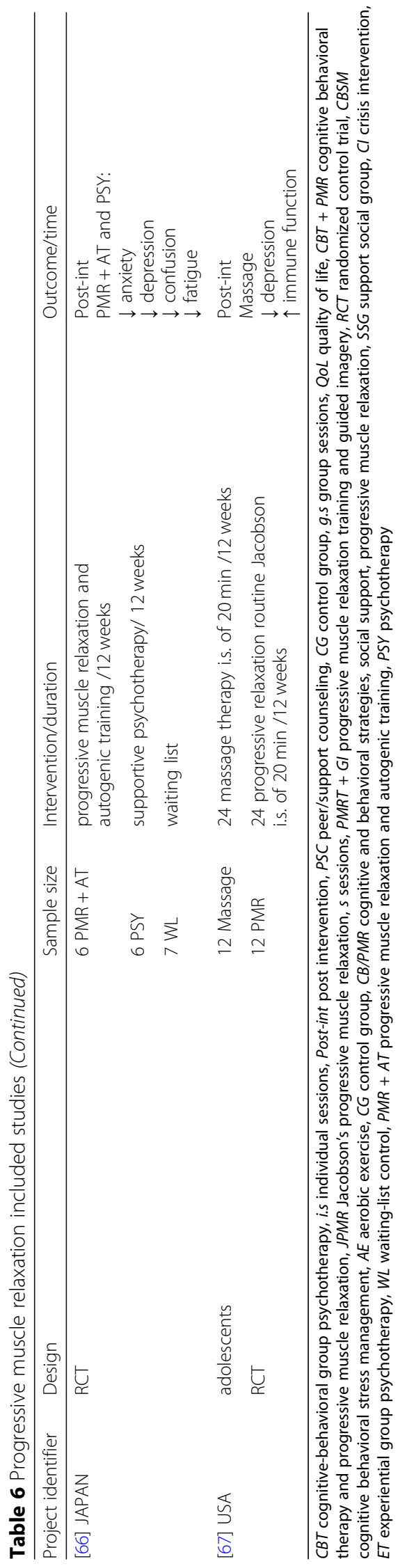




\section{Hypnosis}

We identified 10 documents ( 5 articles, 4 dissertations, and 1 conference abstract), which drew on 8 studies assessing hypnosis in people living with HIV [68-75]. Only two studies used a RCT design [74, 75]. Two other studies [72, 73] used an untreated control group with pretest and posttest design, and the last four [68-71] used a one-group pretest-posttest design (Table 7). (Table 7).

HYP: hypnosis; i.s.: individual sessions; Post-int: post intervention; g.s.: group sessions; QoL: quality of life; CG: control group; WL: wait list; RCT: randomized controlled trial; CON: counseling;

Most of the studies had a small sample size. Only four included more than 15 participants per group [70, 71, $73,75]$. Some studies recruited HIV+ gay men [72, 74] or stable HIV people [70, 73]. Two studies enrolled people living with HIV and pain [68, 71], while two others recruited people living with HIV and dermatitis [75] or pruritus [69]. These studies excluded participants with disabling neurological impairment, drug addiction, or alcoholism [72, 74], as well as those with any other acute or chronic illnesses [70,71, 73] or severe depression [75]. Six studies offered individual hypnosis [68, 69, 71-74] ranging from 1 [73] to 10 sessions [68] that lasted 20-70 min. Two studies [70, 75] consisted of nine 2-h group interventions [70] or sixteen 1.5-h group interventions [75]. All the interventions include of daily self-hypnosis practice at home.

Results of three small NRCTs studies suggested hypnosis had beneficial effects on pain [68], itching [69], and anxiety [72]. Two studies with a one group pretest-posttest design and with samples of more than 15 participants found beneficial results of their intervention on coping [70], pain, and quality of life [71]. The final study we identified assessed individual hypnosis over a three-week period found beneficial results on pain and quality of life 1 month after the end of the intervention [71]. One RCT [75], which assessed sixteen 1.5-h sessions of group hypnosis found positive results on the vitality of

Table 7 Hypnosis included studies

\begin{tabular}{|c|c|c|c|c|}
\hline Project identifier & Design & Sample size & Intervention/duration & Outcomes/time \\
\hline$[68,128]$ USA & $\begin{array}{l}\text { one group pretest-posttest } \\
\text { design }\end{array}$ & $5 \mathrm{HYP}$ & $\begin{array}{l}10 \text { deep breathing, hypnotic induction, pain } \\
\text { relief and sleep suggestions i.s. + twice daily } \\
\text { hypnosis home practice / } 10 \text { weeks }\end{array}$ & $\begin{array}{l}\text { Post-int } \\
\downarrow \text { pain } \\
\downarrow \text { pain medication }\end{array}$ \\
\hline $\begin{array}{l}{[69]} \\
\text { New Zealand }\end{array}$ & $\begin{array}{l}\text { one group pretest-posttest } \\
\text { design }\end{array}$ & $3 \mathrm{HYP}$ & $\begin{array}{l}6 \text { guided muscle relaxation, visualization, } \\
\text { imagery and suggestions to control the } \\
\text { itch i.s. }+30 \text { min hypnosis home practice } / 6 \text { weeks }\end{array}$ & $\begin{array}{l}\text { Post-int } \\
\downarrow \text { itch }\end{array}$ \\
\hline [70] India & $\begin{array}{l}\text { one group pretest-posttest } \\
\text { design }\end{array}$ & $20 \mathrm{HYP}$ & $\begin{array}{l}9 \text { progressive muscular relaxation, } \\
\text { ego strengthening, guided imagery, } \\
\text { visualization, and sensory imagery } \\
\text { conditioning techniques g.s. of } 2 \mathrm{~h}+ \\
\text { hypnosis home practice } / 9 \text { weeks }\end{array}$ & $\begin{array}{l}\text { Post-int } \\
\uparrow \text { coping }\end{array}$ \\
\hline$[71,129]$ USA & $\begin{array}{l}\text { one group pretest-posttest } \\
\text { design }\end{array}$ & $41 \mathrm{HYP}$ & $\begin{array}{l}3 \text { hypnosis i.s of } 70 \text { min }+ \text { self-hypnosis } \\
\text { practice with specific target goals } / 3 \text { weeks }\end{array}$ & $\begin{array}{l}\text { Post-int \& } 7 \text { weeks } \\
\downarrow \text { pain } \\
\uparrow \text { QoL }\end{array}$ \\
\hline \multirow[t]{2}{*}{ [72] USA } & \multirow[t]{2}{*}{$\begin{array}{l}\text { untreated control group with } \\
\text { pretest and posttest design }\end{array}$} & $14 \mathrm{HYP}$ & $\begin{array}{l}6 \text { inner guide techniques, the shamanic } \\
\text { journey technique, mental imagery involving } \\
\text { the immune system i.s. of } 20 \text { min + daily self- } \\
\text { hypnosis home practice / } 6 \text { weeks }\end{array}$ & \multirow[t]{2}{*}{$\begin{array}{l}\text { Post-int } \\
\downarrow \text { anxiety } \\
\uparrow \text { CD4/CD8 ratio }\end{array}$} \\
\hline & & 9 CG & usual care & \\
\hline \multirow[t]{2}{*}{ [73] USA } & \multirow[t]{2}{*}{$\begin{array}{l}\text { untreated control group with } \\
\text { pretest and posttest design }\end{array}$} & $16 \mathrm{HYP}$ & $\begin{array}{l}1 \text { hypnosis and imagery training to relief distress, } \\
\text { to address immune functioning and to impart a } \\
\text { sense of self-efficacy and control i.s. + daily } \\
\text { hypnosis home practice / } 12 \text { weeks }\end{array}$ & \multirow[t]{2}{*}{ Post-int } \\
\hline & & $17 \mathrm{WL}$ & wait list & \\
\hline \multirow[t]{3}{*}{ [74] USA } & \multirow[t]{3}{*}{ RCT } & $19 \mathrm{HYP}$ & $\begin{array}{l}3 \text { stress discussion and hypnosis i.s. of } 45 \mathrm{~min}+\text { daily } \\
\text { hypnosis home practice } / 1 \text { week }\end{array}$ & \multirow[t]{3}{*}{ Post-int } \\
\hline & & $15 \mathrm{CON}$ & 3 stress discussion i.s. of $45 \mathrm{~min}$ & \\
\hline & & $9 \mathrm{CG}$ & usual care & \\
\hline \multirow[t]{2}{*}{ [75] India } & \multirow[t]{2}{*}{$\mathrm{RCT}$} & $90 \mathrm{HYP}$ & $\begin{array}{l}16 \text { progressive muscular relaxation, } \\
\text { ego strengthening, changing of negative } \\
\text { mood set to positive mood set and boosting } \\
\text { up of immune system g.s. of } 90 \text { min + self- } \\
\text { hypnosis home practice/ } 16 \text { weeks }\end{array}$ & \multirow[t]{2}{*}{$\begin{array}{l}\text { Post-int \& } 6 \text { months } \\
\uparrow \text { vitality }\end{array}$} \\
\hline & & 90 CG & usual care & \\
\hline
\end{tabular}


people living with HIV and dermatitis. Another RCT [74] assessing a one-week individual hypnosis intervention does not show significant results. All studies were unblinded to participants and only one [74] was blinded to outcome assessors. Three studies [70, 73, 74] were rated high risk of attrition.

\section{Guided imagery}

We found 10 documents (5 articles and 5 dissertations) drawing on six studies that assessed guided imagery in people living with HIV. Two studies $[79,80]$ used a RCT design, and another [81] had a pilot RCT design. One study [78] used an untreated control group and two others [76, 77] had a one-group pretest-posttest design (Table 8).

Two studies $[76,79]$ had fewer than 15 participants per group and the other studies varied between 20 [77, $80]$ and 30 participants $[78,81]$. They were composed of men [79], women [81], or both genders living with HIV $[76,80]$. Two studies included patients with a variety of diagnoses, such as cancer, AIDS, viral infections or post-surgical recovery. Participants with cognitive impairment or drug or alcohol addictions were excluded.
Four studies [77, 78, 80, 81] offered guided imagery as an individual intervention. One included a single 30-min session of guided imagery [78] and the other three [77, 80, 81] offered one training session followed by daily practice over 5 days to 6 weeks. The other two studies offered 1.5-h sessions of guided imagery in small groups once a week, over a period of six [76] to 8 weeks [79] with daily practice at home.

A very small NRCT $(n=7)$ suggested the existence of the beneficial effects of guided imagery on anxiety and self-control [76]. Two NRCTs studies with sample sizes of more than 15 participants found beneficial effects on anxiety, depression, pain, self-esteem [78] and white blood cell counts [77]. RCTs results show decreased symptoms of depression, fatigue [80], and mood disturbance [79] after relaxation induction and guided imagery was practiced once or twice per day. Results on mood disturbance were maintained a month after the end of the intervention [79]. All studies were unblinded to participants and they did not give information on blinding of outcome assessors. Two studies $[76,79]$ were deemed at a high risk of attrition.

Table 8 Guided imagery included studies

\begin{tabular}{|c|c|c|c|c|}
\hline Project identifier & Design & Sample size & Intervention/duration & Outcome/time \\
\hline [76] USA & $\begin{array}{l}\text { one group pretest-posttest } \\
\text { design }\end{array}$ & $7 \mathrm{GIR}$ & $\begin{array}{l}6 \text { guided imagery and relaxation g.s } \\
\text { of } 1 \mathrm{~h} 30+\text { home practice } / 6 \text { weeks }\end{array}$ & $\begin{array}{l}\text { Post-int } \\
\uparrow \text { self-control } \\
\downarrow \text { anxiety }\end{array}$ \\
\hline$[77,130]$ USA & $\begin{array}{l}\text { one group pretest-post-test } \\
\text { design }\end{array}$ & $20 \mathrm{VT}$ & $\begin{array}{l}1 \text { visualization training with verbal } \\
\text { suggestion and visualization instructions } \\
\text { i.s. of } 30 \mathrm{~min}+\text { daily home practice } / 5 \text { days }\end{array}$ & $\begin{array}{l}\text { Post-int } \\
\uparrow \text { white blood cell count }\end{array}$ \\
\hline \multirow[t]{2}{*}{ [78] USA } & \multirow{2}{*}{$\begin{array}{l}\text { untreated control group with } \\
\text { pretest and posttest design }\end{array}$} & 30 MSG & 1 slow stroke back massage i.s. of $30 \mathrm{~min}$ & \multirow[b]{2}{*}{$\begin{array}{l}\text { Post-int } \\
\text { MSG and GI } \\
\downarrow \text { pain } \\
\downarrow \text { anxiety } \\
\downarrow \text { depression } \\
\uparrow \text { self-esteem }\end{array}$} \\
\hline & & $30 \mathrm{Gl}$ & $\begin{array}{l}1 \text { autogenic induction and guided imagery } \\
\text { i.s. of } 30 \mathrm{~min}\end{array}$ & \\
\hline \multirow[t]{3}{*}{ [79] USA } & \multirow[t]{3}{*}{$\mathrm{RCT}$} & 11 PST & $\begin{array}{l}8 \text { problem solving training g.s. of } 1 \text { h30 }+ \\
\text { homework / } 8 \text { weeks }\end{array}$ & \multirow{3}{*}{$\begin{array}{l}\text { Post-int. \& } 3 \text { moths } \\
\text { RGIT } \\
\downarrow \text { mood disturbance } \\
\downarrow \text { hopelessness }\end{array}$} \\
\hline & & 12 RGIT & $\begin{array}{l}8 \text { relaxation and guided imagery training } \\
\text { g.s. of } 1 \text { h30 + twice daily home practice } / 8 \text { week }\end{array}$ & \\
\hline & & $13 C G$ & control group & \\
\hline \multirow[t]{3}{*}[80,131,132]{ USA } & \multirow[t]{3}{*}{$\mathrm{RCT}$} & $23 \mathrm{Gl}$ & $\begin{array}{l}1 \text { relaxation induction and guided imagery } \\
\text { i.s. of } 22 \mathrm{~min}+\text { daily practice } / 6 \text { weeks }\end{array}$ & \multirow{3}{*}{$\begin{array}{l}\text { Post-int } \\
\text { Gl } \\
\downarrow \text { depression } \\
\downarrow \text { fatigue } \\
\text { PMR } \\
\downarrow \text { depression } \\
\uparrow \text { CD4+ }\end{array}$} \\
\hline & & $22 \mathrm{PMR}$ & $\begin{array}{l}\text { Progressive muscle relaxation home practice } \\
16 \text { weeks }\end{array}$ & \\
\hline & & 24 CG & usual care & \\
\hline \multirow[t]{2}{*}[81,133]{ USA } & \multirow[t]{2}{*}{ pilot RCT } & 30 CSMT & $\begin{array}{l}1 \text { stress, cognitive appraisal process, } \\
\text { coping strategies, guided imagery } \\
\text { i.s. of } 1 \mathrm{~h} 30+\text { home practice } \\
\text { /4 weeks }\end{array}$ & \multirow[t]{2}{*}{$\begin{array}{l}\text { Post-int } \\
\uparrow \text { stress management knowledge }\end{array}$} \\
\hline & & $30 \mathrm{WL}$ & wait list & \\
\hline
\end{tabular}

GIR guided imagery and relaxation, g.s group sessions, Post-int post intervention, $V T$ visualization training, MSG massage, Gl guided imagery, i.s individual sessions, $R C T$ randomized controlled trial, $P S T$ problem solving training, $R G I T$ relaxation and guided imagery training, $P M R$ progressive muscle relaxation, $C G$ control group CSMT computerized stress management training, WL wait list 


\section{Autogenic training, breathing, and neurofeedback}

We found four documents reporting on three studies that assessed autogenic relaxation technique [82], neurofeedback [84], and breathing exercises [83] in people living with HIV. Neurofeedback was assessed using an RCT design in one of these studies [84] and the other two $[82,83]$ used a one-group pretest-posttest design (Table 9). Two of the studies assessed the combination of autogenic training exercises [82] or breathing exercises [83] with physical, mental, and spiritual activities over a seven to eight week period. Neurofeedback was compared to home cranial electrotherapy and to the combination of these two interventions over a 16 -week period.

A study assessing autogenic training in groups over 7 weeks found beneficial results for quality of life in people living with HIV/AIDS both immediately and 8 months after the intervention [82]. A small study $(n=$ 13) assessing a spiritual coping group intervention, which includes breathing exercises, showed an increase in the use of positive spiritual coping and a decrease in symptoms of depression after the intervention in a group of adults living with HIV [83]. Finally, neurofeedback alone or in combination with daily home cranial electrotherapy seemed to decrease symptoms and increase CD4 levels in people living with HIV with CD4 counts between 150 and 650/cc [84]. The RCT was unblinded to participants and this did not give information on blinding of outcome assessors. One study [82] was deemed at high risk of attrition.

\section{Combination of at least three relaxation techniques}

We identified 47 documents (39 articles, 6 dissertations, and 2 conference abstracts) drawing on 20 studies that assessed a combination of at least 3 relaxation techniques with or without other components in people living with HIV [85-104] (Table 10). Most studies (13/20) used an RCT design [91-93, 95-104]. One [94] used a pilot RCT design and collect qualitative data to describe participants' perceptions about the efficacy of the intervention. Four studies [87-90] used an untreated control group with pretest and posttest design and two studies $[85,86]$ had a one-group pretest-posttest design with a very small size sample.

Seven studies [85-87, 91, 92, 95, 96] had less than 15 participants per group. The size of the other studies varied between $40[93,97]$ and over 400 participants [90, 103]. Most recruited stable seropositive people [85-88, 90-93, 95-99, 103]. Six other studies [89, 94, 100, 101, 104] recruited symptomatic seropositive people or women with a cervical lesion [102]. Most studies excluded participants with cognitive impairment, psychiatric disorder, or drug or alcohol addictions. Seven studies $[85-87,91,92,95,96]$ assessed a combination of at least three relaxation techniques, including biofeedback, breathing, progressive muscle relaxation, autogenic training, guided imagery and hypnosis. Most of these interventions (5/7) were offered individually and included home practice (6/7). They were divided into 7 to 20 sessions, lasting between 1 and $2 \mathrm{~h}$, for between 8 and 13 weeks. Seven studies [90, 93, 98-100, 102, 103] assessed the same cognitive-behavioral stress management intervention (CBSM) intervention. In this intervention, participants attended 10 weekly 2.25 -h group sessions (broken down into 90 min of cognitive behavioral (CB) training and $45 \mathrm{~min}$ of relaxation) and were instructed to practice relaxation exercises twice a day between sessions. The relaxation exercises included progressive muscle relaxation, autogenic training, meditation and deep breathing. Four other studies [88, 89, 94, 97] assessed a combination of relaxation techniques, yoga exercises, and/or meditation with cognitive-behavioral strategies. Three of these offered group interventions $[88,89,94]$ and the fourth had individual sessions.

Table 9 Autogenic training, breathing and neurofeedback included studies

\begin{tabular}{|c|c|c|c|c|}
\hline Project identifier & Design & Sample size & Intervention/duration & Outcome/time \\
\hline [82] UK & $\begin{array}{l}\text { one group pretest-post-test } \\
\text { design }\end{array}$ & 50 AT & $\begin{array}{l}7 \text { diet, nutrition, supplements, exercise, } \\
\text { safer sex, recreational drugs, spiritual experiences, } \\
\text { autogenic training g.s. of } 2 \mathrm{~h}+\text { autogenic exercises } \\
\text { home practice } / 7 \text { weeks }\end{array}$ & $\begin{array}{l}\text { Post-int \& } 8 \text { months } \\
\uparrow \text { quality of life }\end{array}$ \\
\hline [83] USA & $\begin{array}{l}\text { one group pretest-post-test } \\
\text { pilot design }\end{array}$ & 13 SCGI & $\begin{array}{l}8 \text { spirituality, mental and physical health, } \\
\text { personal goals, relaxation breathing exercise } \\
\text { g.s. of } 1 \text { h15 / } 8 \text { weeks }\end{array}$ & $\begin{array}{l}\text { Post-int } \\
\uparrow \text { positive spiritual coping } \\
\downarrow \text { depression }\end{array}$ \\
\hline \multirow[t]{4}{*}[84,134]{ USA } & \multirow[t]{4}{*}{ RCT } & $10 \mathrm{NFB}$ & 32 neurofeedback i.s. of $20 \mathrm{~min} / 16$ weeks & \multirow{4}{*}{$\begin{array}{l}\text { Post-int } \\
\text { NFB and NFB }+ \text { HDCE } \\
\downarrow \text { symptoms } \\
\uparrow \text { CD4+ }\end{array}$} \\
\hline & & $10 \mathrm{HDCE}$ & $\begin{array}{l}20 \text { min daily cranial electrotherapy home } \\
\text { practice } / 16 \text { weeks }\end{array}$ & \\
\hline & & $10 \mathrm{NFB}+\mathrm{HDCE}$ & $\begin{array}{l}32 \text { neurofeedback i.s. of } 20 \mathrm{~min}+20 \mathrm{~min} \\
\text { daily cranial electrotherapy home practice } / 16 \text { weeks }\end{array}$ & \\
\hline & & $10 \mathrm{WL}$ & wait list & \\
\hline
\end{tabular}

$A T$ autogenic training, g.s group sessions, Post-int post intervention, SCGl spiritual coping group intervention, $R C T$ randomized control trial, NFB neurofeedback, $H D C E$ home daily cranial electrotherapy, NFB + HDCE neurofeedback and home daily cranial electrotherapy, WL wait list, i.s individual sessions 


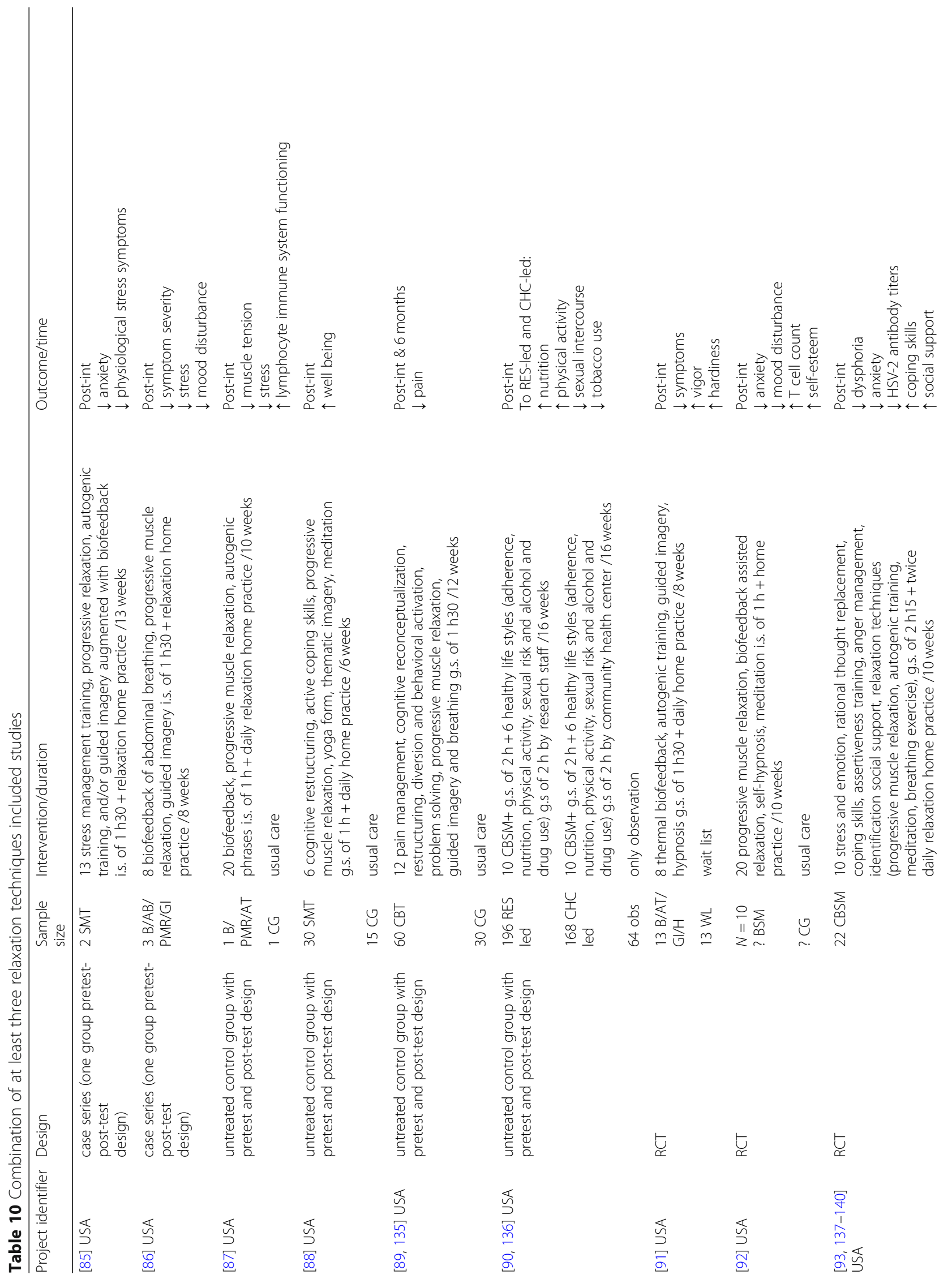




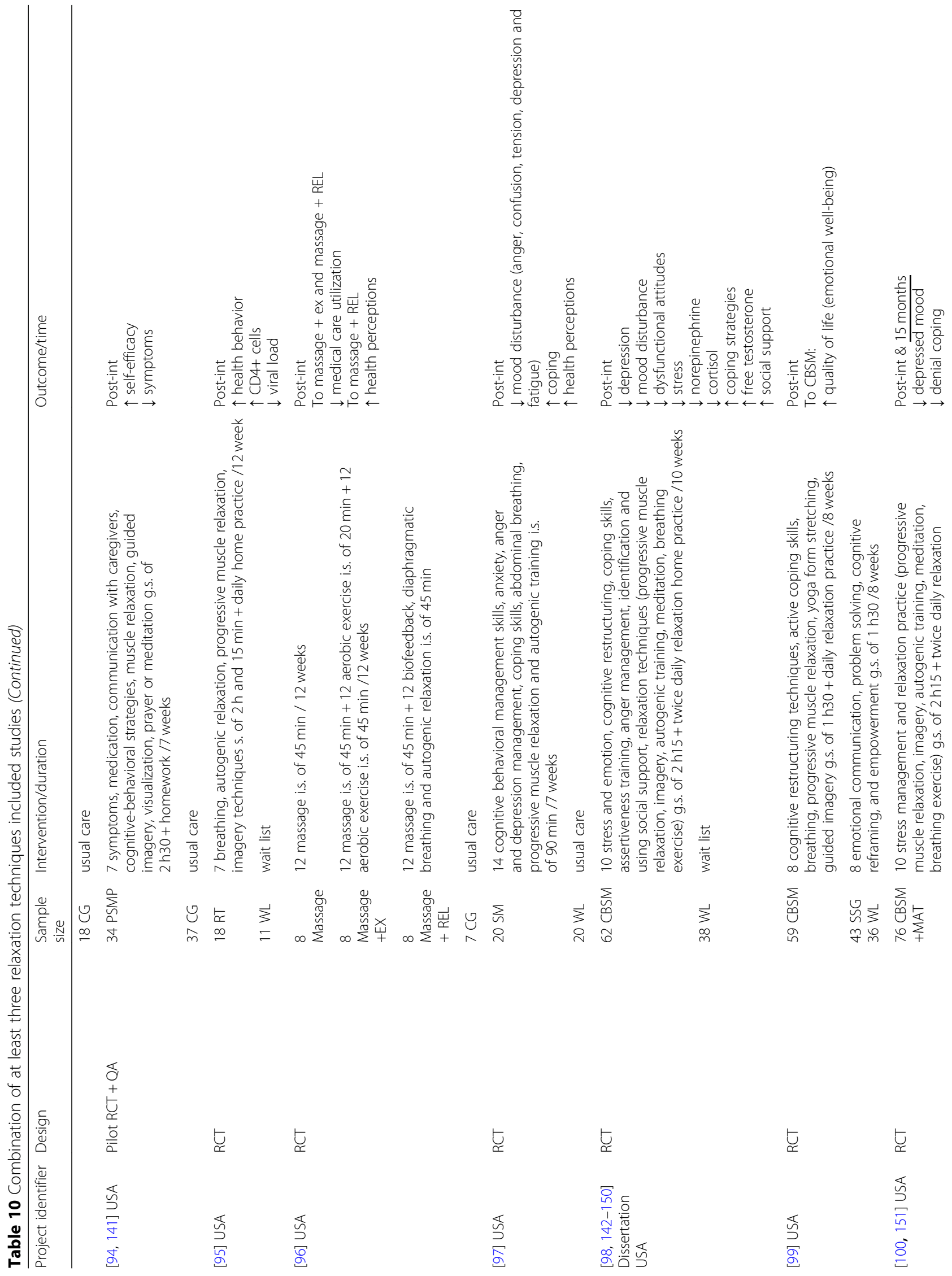




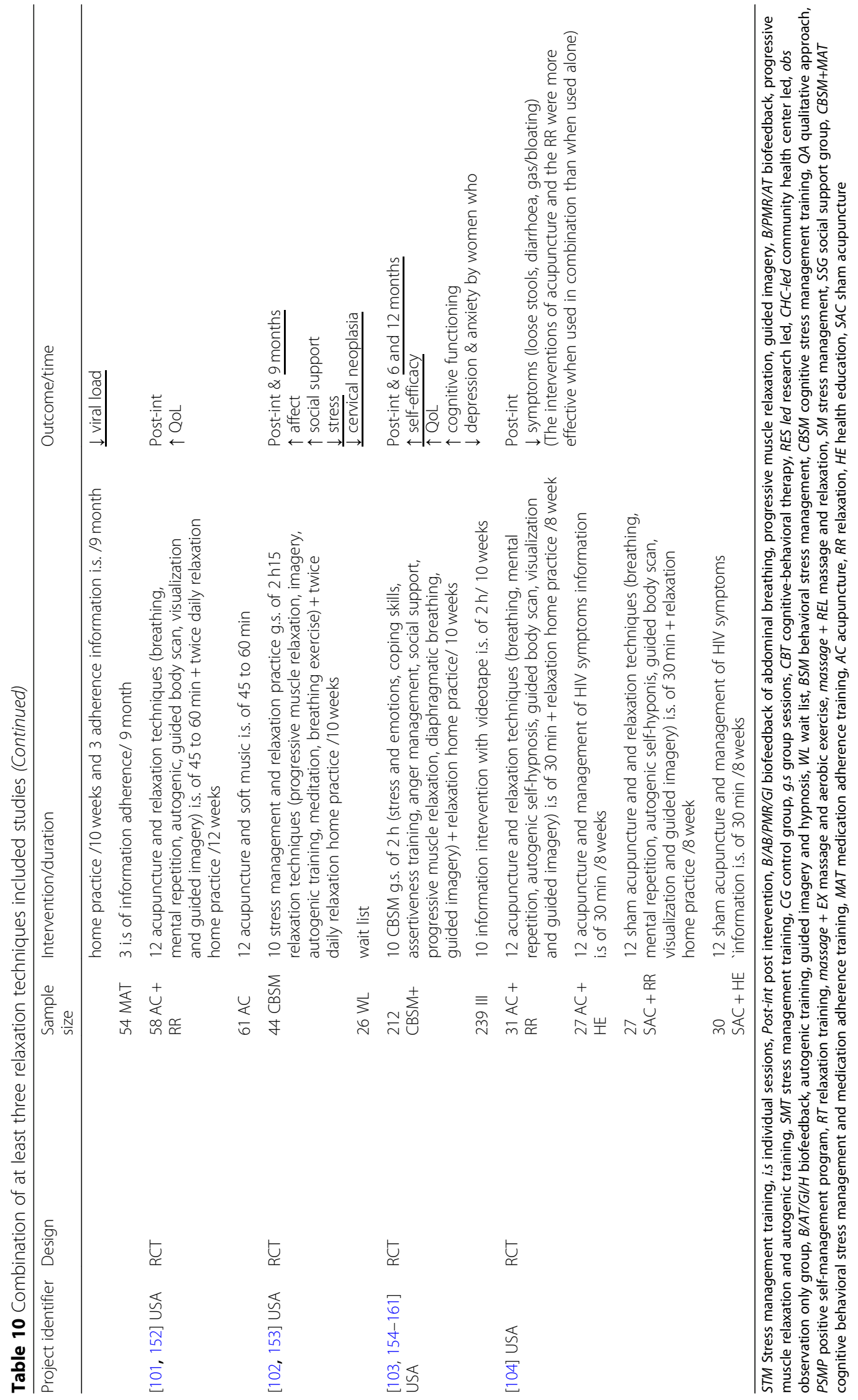


Interventions consisted of six to twelve $1-2.5 \mathrm{~h}$ sessions over 6-12 weeks. Two interventions included the relaxation practice at home. Two studies [101, 104] assessed a set of relaxation techniques used in combination with acupuncture. These individually offered interventions included 7-20 sessions over $6-16$ weeks with relaxation exercises at home.

Three NRCTs, which had two to three participants [85-87] and assessed a combination of at least three relaxation techniques without other components, found beneficial results on anxiety, mood disturbance, stress, symptom severity and lymphocytes count. Four small RCTs $[91,92,95,96]$ also examining this type of intervention showed beneficial effects post intervention on general symptoms, energy, confidence, anxiety, distress, health-related behaviors, use of health services, viral load and CD4 counts. However, these studies were small with fewer than 15 participants per group.

One NRCT [90] found that CBSM intervention in women living with HIV was associated with improvement in behavioral outcomes, and these outcomes were comparable between groups led by research and community health centers. Six RCTs [93, 98-100, 102, 103] that assessed CBSM showed beneficial results on anxiety, distress, coping abilities, social support, stress, cortisol and testosterone levels, cognitive function, perceived self-efficacy, cervical neoplasia, quality of life and viral load. One of these studies [93] found that the number of weeks an individual practiced relaxation at least once was significantly correlated with changes in depression and anxiety. Three of these studies showed positive results of the interventions over the long-term (6-15 months) on perceived self-efficacy [103], stress, cervical neoplasia [102] and viral load [100].

Two pretest and posttest studies $[88,89]$ with an untreated control group found that a combination of relaxation techniques and meditation exercises with cognitive-behavioral strategies improved wellbeing [88] and decreased pain [89]. Increased weekly practice was found to be significantly related to increased positive impact on stress, higher quality of life, more frequent use of problem-focused coping, less avoidant thinking and uncertainty [88]. Effects on pain were long-term (more than 6 months) [89]. Two RCTs [94, 97] that assessed a combination of relaxation techniques and meditation exercises with cognitive-behavioral strategies showed beneficial results on symptoms, mood disturbance, perceived self-efficacy, coping and health perceptions. Finally, two RCTs that assessed a combination of relaxation techniques, such as breathing, autogenic training, body-scan exercise and guided imagery with acupuncture found beneficial results on quality of life [101] and general symptoms [104]. All but two studies [101, 104] were unblinded to participants; one study [100] was unblinded to participants, but blinded to outcome assessors. Eleven studies [86, 89-91, 93, 95, 96, 98, 99, 102, 103] were rated at high risk of attrition (Table 3).

\section{Unspecified relaxation}

We found 10 documents ( 9 articles and 1 dissertation) on 8 studies [105-112] that assessed cognitive-behavioral strategies with a relaxation technique without indicating the type of relaxation technique used (Table 11). They consisted of six RCTs [107-112], one untreated control group with pretest and posttest design [106], and one group pretest-posttest design [105].

Three studies had fewer than 15 participants per group $[105,109,112]$. The sample size of the other studies varied between 50 [106] and 120 [110]. Participants were heterosexual [112] and homosexual or bisexual men [106] living with HIV $[107,109,111]$. Two studies [105, 110] recruited homosexual or bisexual men living with HIV and depression. One study recruited adults with perceived risk for HIV infection [108]. Most studies excluded participants with cognitive impairment, major medical or mental illness, and current alcohol or drug use. Most interventions (5/8) were offered to groups [106, 107, 110-112]. They consisted of between 3 and 22 meetings varying in length between 45 -min and $2.5 \mathrm{~h}$ and held between 3 and 16 weeks. Two interventions $[110,111]$ added 5 or 6 boost sessions over a 912-month period.

Two NRCTs studies $[105,106]$ showed positive results on mood, coping, and adherence. Six RCTs [107-112] found beneficial results on reducing the number of sexual partners, anxiety, depression, mood and stress, and improving lymphocyte count and quality of life. Two of the studies did not describe attrition [107, 109] and the rest, which presented attrition rates between 14 and $20 \%$, did not consider losses in their analysis. All studies were unblinded to participants and did not give any information on blinding of outcome assessors. Four studies $[105,108,110,112]$ were rated as a high risk of attrition.

\section{Discussion}

The amount of research on mind-body practices in people living with HIV over 30 years varies widely depending on the practice. Data from these studies show an increasing research interest over the last 5 years for these practices (particularly meditation and yoga) in the HIV population. Research on hypnosis also seems to have increased slightly in recent years, while the number of studies on progressive muscle relaxation and guided imagery appear to be decreasing. However, research on a combination of least three relaxation techniques seems to remain stable, 
Table 11 Unspecified relaxation included studies

\begin{tabular}{|c|c|c|c|c|}
\hline $\begin{array}{l}\text { Project } \\
\text { identifier }\end{array}$ & Design & $\begin{array}{l}\text { Sample } \\
\text { size }\end{array}$ & Intervention/duration & $\begin{array}{l}\text { Outcome/ } \\
\text { time }\end{array}$ \\
\hline [105] USA & $\begin{array}{l}\text { case series (one group } \\
\text { pretest-post-test } \\
\text { design) }\end{array}$ & $\begin{array}{l}4 \text { CBT- } \\
\text { AD }\end{array}$ & $\begin{array}{l}8 \text { behavioral activation, cognitive restructuring, problem solving, adherence } \\
\text { tools, relaxation i.s. }\end{array}$ & $\begin{array}{l}\text { Post-int } \\
\downarrow \text { depression } \\
\uparrow \text { adherence }\end{array}$ \\
\hline \multirow[t]{2}{*}{ [106] USA } & \multirow[t]{2}{*}{$\begin{array}{l}\text { untreated control group with } \\
\text { pretest and post-test design }\end{array}$} & $\begin{array}{l}N=50 \\
? S M G\end{array}$ & $\begin{array}{l}10 \text { stress management techniques, relaxation training, problem-solving skills } \\
\text { and effective coping styles g.s. of } 2 \mathrm{~h} / 10 \text { weeks }\end{array}$ & \multirow{2}{*}{$\begin{array}{l}\text { Post-int } \\
\uparrow \text { mood } \\
\uparrow \text { coping }\end{array}$} \\
\hline & & ?CG & usual care & \\
\hline \multirow[t]{2}{*}{ [107] USA } & \multirow[t]{2}{*}{$\mathrm{RCT}$} & $\begin{array}{l}N=64 \\
? S M G\end{array}$ & $\begin{array}{l}8 \text { systematic relaxation, health habit change, skills for managing stress g.s. of } \\
2 \mathrm{~h} / 8 \text { weeks }\end{array}$ & \multirow{2}{*}{$\begin{array}{l}\text { Post-int } \\
\downarrow \text { sexual } \\
\text { partners }\end{array}$} \\
\hline & & $? \mathrm{WL}$ & wait-list & \\
\hline \multirow[t]{3}{*}{ [108] USA } & \multirow[t]{3}{*}{$\mathrm{RCT}$} & $32 \mathrm{C}$ & 1 tailored counseling i.s & \multirow{3}{*}{$\begin{array}{l}3 \text { months } \\
\text { To C + SPT + R } \\
\downarrow \text { anxiety } \\
\downarrow \text { psychiatric } \\
\text { symptoms }\end{array}$} \\
\hline & & $\begin{array}{l}31 C+ \\
V+R\end{array}$ & $\begin{array}{l}3 \text { counseling, information video about HIV and relaxation (abdominal } \\
\text { breathing, imaging i.s. of } 45 \text { min } / 3 \text { weeks }\end{array}$ & \\
\hline & & $\begin{array}{l}40 C+ \\
S P T+R\end{array}$ & $\begin{array}{l}6 \text { tailored counseling, cognitive behavioral treatment for depression, stress } \\
\text { and anxiety (education, personal control, training to manage challenging } \\
\text { situations) and systematic relaxation i.s. of } 1 \mathrm{~h} / 6 \text { weeks }\end{array}$ & \\
\hline \multirow{2}{*}{$\begin{array}{l}{[109,162]} \\
\text { South- } \\
\text { Africa }\end{array}$} & \multirow[t]{2}{*}{$\mathrm{RCT}$} & $14 \mathrm{EG}$ & $\begin{array}{l}8 \text { cognitive-behavioral therapy, aerobic exercise, biofeedback-assisted } \\
\text { relaxation i.s. of } 1 \text { h30 + relaxation home practice } / 8 \text { weeks }\end{array}$ & \multirow{2}{*}{$\begin{array}{l}\text { Post-int } \\
\downarrow \text { depression } \\
\uparrow \text { mood } \\
\uparrow \text { lymphocyte } \\
\text { counts }\end{array}$} \\
\hline & & $12 C G$ & $\begin{array}{l}8 \text { individual counseling (psychological and medical aspects of the AIDS) i.s. of } \\
1 \text { h30 / } 8 \text { weeks }\end{array}$ & \\
\hline \multirow[t]{3}{*}{$\begin{array}{l}{[110,163]} \\
\text { USA }\end{array}$} & \multirow[t]{3}{*}{$\mathrm{RCT}$} & 46 CET & $\begin{array}{l}10 \text { stress management, coping strategies, social support, relaxation g.s. of } 1 \\
\text { h30 on } 10 \text { weeks }+6 \text { boosters stress management sessions } / 12 \text { months }\end{array}$ & \multirow{3}{*}{$\begin{array}{l}\text { Post-int \& } 6 \\
\text { and } 12 \\
\frac{\text { months }}{\downarrow \text { stress }} \\
\frac{\downarrow \text { burnout }}{\downarrow \text { anxiety }} \\
\uparrow \text { coping self- } \\
\text { efficacy }\end{array}$} \\
\hline & & 44 HIV-I & $\begin{array}{l}10 \text { HIV information g.s. of } 1 \text { h30 on } 10 \text { weeks }+6 \text { boosters information } \\
\text { sessions } / 12 \text { months }\end{array}$ & \\
\hline & & $31 \mathrm{WL}$ & wait-list & \\
\hline \multirow[t]{2}{*}{$\begin{array}{l}{[111]} \\
\text { Netherland }\end{array}$} & \multirow[t]{2}{*}{$\mathrm{RCT}$} & $44 \mathrm{Gl}$ & $\begin{array}{l}17 \text { stress management, coping skills, social support, relaxation, written HIV } \\
\text { information g.s. of } 2 \text { h30 on } 16 \text { weeks }+5 \text { stress management, coping skills, } \\
\text { social support, relaxation g.s. } / 9 \text { months }\end{array}$ & \multirow[t]{2}{*}{$\begin{array}{l}\text { Post-int } \\
\uparrow \text { coping }\end{array}$} \\
\hline & & $41 \mathrm{EC}$ & written hiv information & \\
\hline \multirow[t]{2}{*}{ [112] China } & $\mathrm{RCT}$ & $6 \mathrm{CBP}$ & $\begin{array}{l}7 \text { coping skills, social support, cognitive restructuring, behavior change } \\
\text { strategies, relaxation, g.s. of } 2 \mathrm{~h}+\text { daily relaxation home practice/ } 7 \text { weeks }\end{array}$ & \multirow{2}{*}{$\begin{array}{l}\text { Post-int } \\
\uparrow \text { mood } \\
\uparrow \text { QoL }\end{array}$} \\
\hline & & $7 \mathrm{WL}$ & wait list & \\
\hline
\end{tabular}
CBT-AD cognitive behavioral technique and adherence tools, i.s individual sessions, Post-int post intervention, SMG stress management group, g.s group sessions, $C G$ control group, $W L$ wait list, $C$ counseling, $C+V+R$ counseling, video and relaxation, $C+S P T+R$ counseling, stress prevention training and relaxation, $E G$ experimental group, CET coping effectiveness training, HIV-I information about HIV, GI group intervention, EC educational control, CBP cognitive behavioral program

with three or four studies for every five-year period since 1990 .

About two-thirds of the studies examining a combination of at least three relaxation techniques, yoga, and Tai Chi/Qigong, and half of the studies on progressive muscle relaxation, meditation, and guided imagery used a RCT design. Most studies were conducted on general samples of people living with HIV and excluded participants with any history of drug or alcohol abuse, current psychiatric major disorders, or cognitive impairment. Half of the studies on hypnosis and on progressive muscle relaxation and some of the studies on yoga, meditation or on a combination of at least three relaxation techniques recruited participants living with HIV who have depression, anxiety, pain or other symptoms. Three studies-on mindfulness
[45], on yoga [40], and on progressive muscle relaxation [67] - were conducted with adolescents to assess the effects of these techniques on their psychological wellbeing. Two studies-on yoga and on meditation-were conducted on drug users to assess the effects of these interventions on quality of life [42] and on abstinence [51]. Two studies from the 1990s recruited people with perceived risk for HIV [108] or who were unaware of their HIV antibody status [63] in the goal of examining relaxation's effectiveness in reducing emotional distress after testing. Interventions were mainly conducted in groups with the exception of hypnosis, guided imagery, and neurofeedback, which were performed individually. All studies on hypnosis and most studies on meditation, guided imagery, and a combination of least three relaxation 
techniques consisted of individual daily practice at home. The duration of these interventions varied between a few hours and 1 year. The most frequent duration for yoga, meditation, and a combination of at least three relaxation techniques was 8 weeks. The most frequent length of progressive muscle relaxation interventions was 12 weeks, and Tai Chi/Qigong interventions lasted between 8 and 12 weeks. The duration of hypnosis and guided imagery was extremely variable.

In this systematic scoping review, the results of three rigorous RCTs show that meditation and, specifically mindfulness-based stress reduction (MBSR) decrease physical symptoms and ARV side effects $[56,57]$ and improve the psychological state and mindfulness of people living with HIV [58]. These results are confirmed by a systematic review [164] of MBSR for people living with HIV. Indeed, we found that the studies reviewed offer preliminary support for positive effects of MBSR on health and emotional wellbeing. A systematic review [165] of meditation in chronic disease found that most studies (24/45) were based on MBSR and this intervention improves symptoms of anxiety and depression and decreases symptoms in chronic diseases. An overview of systematic reviews and meta-analyses of RCTs using MBSR-based interventions [166] found that MBSR improves symptoms of depression, anxiety and stress, as well as the quality of life and physical functioning in individuals with various conditions, as compared to wait-list control and usual care groups. A well designed RCT study included in this scoping review [54] suggests that repeating a mantra for 5 weeks can decrease psychological distress in people with HIV. More studies are needed to confirm these findings on mantras and identify the effects of other types of meditation, such as transcendental meditation, in people living with HIV.

Two well-designed RCTs [94, 97] assessing a combination of at least three relaxation techniques, with meditation exercises, and cognitive-behavioral strategies show beneficial results on symptoms, mood disturbance, cognitive functioning, and health perceptions. A rigorous RCT [100] show that a cognitive-behavioral stress management (CBSM) program, a combination of relaxation techniques (progressive muscle relaxation, autogenic training and deep breathing) and cognitive behavioral training, had positive effects on depression and viral load. Four others RCTs with some major methodological limitations $[93,98$, $102,103]$ found that a CBSM program seemed decrease anxiety and depression and to increase the cognitive functioning, quality of life, and social support of people living with HIV. Studies with other populations show results similar to CBSM on physical symptoms [167], psychological symptoms [168, 169], and quality of life [169]. However, it should be noted that this type of intervention is very complex and therefore not very accessible.
Furthermore, much of the intervention is based on the practice of these relaxation techniques. In this regard, two well-designed RCTs $[101,104]$ examining the effects of adding a combination of relaxation techniques (deep breathing, autogenic training, body-scan exercise and guided imagery) to usual acupuncture found that this combination had more effects on decresing gastrointestinal symptoms and increasing quality of life than acupuncture alone. Four small RCTs [91, 92, 95, 96] assessing a combination of at least three different relaxation techniques suggest positive effects on symptoms, anxiety, distress, health behavior, viral load and CD4 count in people living with HIV. However, further rigorous research with larger sample sizes are necessary to assess the effect on people living with HIV of the combination of at least three relaxation techniques without other strategies.

Due to the major methodological limitations of these studies, there is little evidence of the effectiveness of each relaxation technique used separately. Some RCT included in this scoping review (but holding high risk of bias in some domains) found that progressive muscle relaxation $[64,65,126]$ improved the psychological state of people living with HIV. The results of two NRCT studies $[68,71]$ suggest that hypnosis decreased pain and improved quality of life in this population. The findings of two NRCT $[76,78]$ and two small RCTs $[79,80]$ suggest that guided imagery had some effect on mood improvement. And an NRCT study on autogenic training [82] and a RCT on neurofeedback [84] show positive effects on the quality of life and the symptoms of people living with HIV. These results are consistent with those provided by some systematic reviews of progressive muscle relaxation [19, 170, 171], hypnosis [172], guided imagery [173], autogenic training [174], and neurofeedback [175] in a variety of populations. Except neurofeedback, which requires specialized equipment and professionals, all the relaxation techniques studied are very accessible, inexpensive, and easy to practice following brief training. Moreover, these relaxation practices involve low emotional and physical risk and allow patients to take a more active role in their treatment. Further research is necessary to prouve the effects of these relaxation techniques used separately by people living with HIV.

A rigorously led RCT [20] found that systolic and diastolic blood pressures improved more in yoga group after 20 weeks of supervised Asthange Vinyasa practice and home practice than in the standard of care group of HIV-infected adults with displaying cardiovascular disease risk factors. A systematic review [176] concluded that yoga appears to be promising in modifying risk factors for cardiovascular disease and metabolic syndrome in adults. Furthermore, results from two one-group studies $[36,37]$ and from four RCT with some important 
methodological limitations suggest that this practice could be used to decrease psychological distress [40, 42], increase quality of life $[38,41]$, and improve the immune system [40] in people living with HIV. Similary, a systematic review of a randomized control trial on the effects of yoga on mood and stress-reduction measures in diverse populations [177] suggests that yoga leads to better regulation of the sympathetic nervous system and the hypothalamic-pituitary-adrenal system, as well as to decrease in symptoms of depression and anxiety. However, more rigorous RCTs are needed to confirm these findings on the emotional and immunological status and quality of life and to identify the effects of long-term yoga practice in people living with HIV.

There is little evidence of the effectiveness of Tai Chi and Qigong in people living with HIV. A well-designed RCT included in this systematic scoping review [31] found that a 10-weeks group Tai Chi intervention for decreased the use of emotion-focused coping strategies and increase lymphocyte proliferation function in people living with HIV. One study with a one group pretest-posttest design suggested that Qigong may improve emotional and immunological status in this population [32]. These results seem to be consistent with those obtained from various populations living with chronic disease. In fact, results from systematic reviews show that Tai Chi improves physical performance in people living with cancer, osteoarthritis, heart failure and chronic obstructive pulmonary disease [178], as well as with symptoms of depression and anxiety in elderly and other populations living with chronic disease [16]. The practice of Qigong also seems to have a positive effect on the severity of depressive symptoms in people living with chronic disease [179]. Additionally, some studies reported improvements in a number of immune-related blood markers among Qigong and Tai Chi practitioners [180], but more studies are needed to confirm these results on the immune system [181]. For people living with HIV, more rigorous RCT are needed to confirm the results of Tai Chi and Qigong on physical, emotional, and immunological status as well as on quality of life.

\section{Strengths and limitations}

The Arksey \& O'Malley framework [22] provides an appropriate methodological foundation for scoping reviews (Levac \& al., 2010). The combination of this method with Cochrane recommendations for systematic reviews of interventions [25] allowed us to consider the risk of bias in the interpretation of study results and to specify the steps of research, identification, data extraction and synthesis. The combination of these methods led us to call the review "a systematic scoping review." An additional strength of this type of review is to present the results according to the type of mind-body practice. This strategy makes it possible to better understand evidence-based results available for each type of mind-body practice and to identify the lack of existing knowledge for each practice.

Nonetheless, this scoping systematic review also has some limitations. This review includes studies published only until October 2015. Although a thorough search strategy was used, there may still be gaps from unpublished data mainly from studies from 90s. Some trials did not provide descriptions of the nature of the mind-body practices tested. The limitations of evidence-based results from the studies included in this review must also be added. Only slightly over half of the studies (47/84) used an RCT design. All but two studies were unblinded to participants and only five used assessors blinded to treatment allocation. Another important problem is the high attrition rate $(33 / 84)$ and a success rate for interventions that fell below $60 \%$ for several studies. Furthermore, most studies did not address incomplete data adequately and excluded dropouts from the final analysis. Finally, some studies were too small to draw any firm conclusions. Risk of bias, however, tended to be lower in the studies published more recently, as compared to older studies.

\section{Conclusions}

\section{Implications for clinical practice}

In our systematic scoping review, we showed that some mind-body practices have encouraging results in improving physical and psychological wellbeing and health in people living with HIV. The practices for which there is better evidence are mindfulness, a combination of at least three relaxation techniques with cognitive behavioral strategies, and yoga. An 8-weeks of mindfulness-based stress reduction (MBSR) program could be suggested to decrease physical symptoms and ARV side effects, and improve the psychological state of people living with HIV. A combination of at least three relaxation techniques and cognitive behavioral strategies could be used to decrease physical and psychological symptoms, and increase quality of life and health in this population. A combinaison of deep breathing, autogenic training, body scan, guided imagery, and acupuncture could be suggested to people living with HIV and gastrointestinal symptoms. Asthange Vinyasa practice could be recommended to lower blood pressure in pre-hypertensive people living with HIV who also display mild-to-moderate cardiovascular disease risk factors. Tai Chi, Qigong, and relaxation techniques used separately may also contribute to the improvement of the physical and psychological condition of people living with HIV, but more rigorous studies are necessary to confirm these results in people living with HIV. 


\section{Implications for research}

Future clinical trials should focus on rigorous study design to minimize bias risks and enhance validity. Although it is difficult with this type of intervention to blind participants, the outcome assessors should be blinded to treatment allocation. All randomized participants should be included in their analysis and intention-to-treat analysis should be used to avoid overoptimistic estimates of the efficacy of interventions [182]. Adhering more closely to CONSORT [183] guidelines for good reporting of clinical trials would considerably help increase the amount of available data. Follow-up on the evaluation of mind-body practices would be important to identify required dosage and modality of interventions for people living with HIV and to accurately determine the contribution of these practices to the health of this population.

\section{Abbreviations}

AIDS: Acquired immune deficiency syndrome; ART: Antiretroviral therapy; CBSM: Cognitive-behavioral stress management intervention;

CHA: Complementary health approaches; HIV: Human immunodeficiency virus; MBSR: Mindfulness-based stress reduction; NRCT: Non-randomized controlled trials; RCT: Randomized controlled trials

\section{Acknowledgements}

The authors would like to thank and acknowledge Ms. Simonetta Mulatero and Ms. Zoë Blowen-Ledoux for the linguistic revision of the manuscript.

\section{Funding}

The publication charges for this article have been funded by a Planning and Dissemination Grants from Canadian Institutes of Health Research, with the grant number PCS155284.

\section{Availability of data and materials}

Most data generated and/or analyzed in the current study are included in this published article. Other data are available from the corresponding author on reasonable request.

\section{Authors' contributions}

PRG conceived the protocol for this scoping systematic review in collaboration with MPG and JC. MD developed and performed the search strategy and built an Endnote database. PRG and SC undertook independent selection of articles from the Endnote database. PRG and MPG undertook data extraction and risk of bias assessment. PRG and JFA were involved in the data synthesis stage. All authors contributed to the development of this manuscript and approved its final version.

Ethics approval and consent to participate

Not applicable.

\section{Consent for publication}

Not applicable.

\section{Competing interests}

The authors declare that they have no competing interests.

\section{Publisher's Note}

Springer Nature remains neutral with regard to jurisdictional claims in published maps and institutional affiliations.

\section{Author details}

${ }^{1}$ Faculty of Nursing, Université de Montréal, Montréal, Canada. ${ }^{2}$ Research Center of the Centre Hospitalier de l'Université de Montréal, Montréal, Canada. ${ }^{3}$ Faculty of Nursing, Université Laval, Québec, Canada. ${ }^{4}$ Research Center of the Centre Hospitalier Universitaire, Québec, Canada. ${ }^{5}$ Faculty of Medicine, Aix Marseille Université, Marseille, France. ${ }^{6}$ University Institute on
Addiction, Montreal-Island-South-Center Integrated University Health and Social Services Centre, Montréal, Canada. ${ }^{7}$ Faculty of Medicine and Health Sciences, Université de Sherbrooke, Longueuil, Canada. ${ }^{8}$ Library, Université Laval, Québec, Canada.

Received: 27 February 2018 Accepted: 15 April 2019

Published online: 11 June 2019

\section{References}

1. Hoogbruin A. Complementary and alternative therapy (CAT) use and highly active antiretroviral therapy (HAART): current evidence in the literature, 2000-2009. J Clin Nurs. 2011;20(7-8):925-39.

2. Thorpe RD. 'Doing' chronic illness? Complementary medicine use among people living with HIV/AIDS in Australia. Sociol Health IIIn. 2009;31(3):37589.

3. Trickey, A., et al., Survival of HIV-positive patients starting antiretroviral therapy between 1996 and 2013: a collaborative analysis of cohort studies. The Lancet HIV, 2017.

4. Ezeamama $A E$, et al. Depressive and anxiety symptoms predict sustained quality of life deficits in HIV-positive Ugandan adults despite antiretroviral therapy: a prospective cohort study. Medicine (Baltimore). 2016;95(9):e2525.

5. Herrmann S, et al. HIV-related stigma and physical symptoms have a persistent influence on health-related quality of life in Australians with HIV infection. Health Qual Life Outcomes. 2013;11:56.

6. Corless IB, et al. The impact of stressful life events, symptom status, and adherence concerns on quality of life in people living with HIV. J Assoc Nurses AIDS Care. 2013;24(6):478-90.

7. Kelso-Chichetto NE, et al. Complementary and alternative medicine use for HIV Management in the State of Florida: medical monitoring project. J Altern Complement Med. 2016;22(11):880-6.

8. Braun LA, et al. Complementary medicine use by people living with HIV in Australia - a national survey. Int J STD AIDS. 2016;27(1):33-8.

9. Lorenc A, Robinson N. A review of the use of complementary and alternative medicine and HIV: issues for patient care. AIDS Patient Care STDs. 2013:27(9):503-10.

10. Littlewood RA, Vanable PA. A global perspective on complementary and alternative medicine use among people living with HIV/AIDS in the era of antiretroviral treatment. Curr HIV/AIDS Rep. 2011;8(4):257-68.

11. Shedlin MG, et al. Use of complementary and alternative medicines and supplements by Mexican-origin patients in a U.S.-Mexico border HIV clinic. J Assoc Nurses AIDS Care. 2013;24(5):396-410.

12. National Center for Complementary and Integrative Health. Complementary, Alternative, or Integrative Health: What's in a name? . 2016. Available from: https://nccih.nih.gov/health/integrative-health. [cited 2018 01/25].

13. Bormann JE, Uphold CR, Maynard C. Predictors of complementary/ alternative medicine use and intensity of use among men with HIV infection from two geographic areas in the United States. J Assoc Nurses AIDS Care. 2009:20(6):468-80.

14. McDonald K, Slavin S. My body, my life, my choice: practices and meanings of complementary and alternative medicine among a sample of Australian people living with HIV/AIDS and their practitioners. AIDS Care. 2010;22(10): 1229-35.

15. National Center for Complementary and Alternative Medicine. What is complementary and altrnative medicine? 2011. Available from: http:// nccam.nih.gov/health/whatiscam. [cited 2011].

16. Wang F, et al. The effects of tai chi on depression, anxiety, and psychological well-being: a systematic review and meta-analysis. Int J Behav Med. 2014;21(4):605-17.

17. Zheng $W$, et al. Tai chi for schizophrenia: a systematic review. Shanghai Arch Psychiatry. 2016;28(4):185-94.

18. Desveaux L, et al. Yoga in the Management of Chronic Disease: a systematic review and meta-analysis. Med Care. 2015;53(7):653-61.

19. Jorm AF, Morgan AJ, Hetrick SE. Relaxation for depression. Cochrane Database Syst Rev. 2008;(4):Cd007142.

20. Cade T, et al. Yoga lifestyle intervention reduces blood pressure in HIVinfected adults with cardiovascular disease risk factors. HIV Med. 2010;11(6): 379-88

21. Chhatre $\mathrm{S}$, et al. Effects of behavioral stress reduction transcendental meditation intervention in persons with HIV. AIDS Care. 2013;25(10):1291-7.

22. Arksey H, O'Malley L. Scoping studies: towards a methodological framework. Int J Soc Res Methodol. 2005;8(1):19-32. 
23. Mays N, Robert E, Popay J. Synthesising research evidence. In: Fulop N, et al. , editors. Studying the organisation and delivery of health services: Research methods. London: Routledge; 2001.

24. Grant MJ, Booth A. A typology of reviews: an analysis of 14 review types and associated methodologies. Health Inf Libr J. 2009;26(2):91-108.

25. Higgins JPT, Green S. Cochrane Handbook for Systematic Reviews of Intervention. Version 5.1.0 [updates March 2011]; 2011.

26. Liberati $A$, et al. The PRISMA statement for reporting systematic reviews and meta-analyses of studies that evaluate healthcare interventions: explanation and elaboration. BMJ. 2009:339:b2700.

27. Tricco, A., S. Straus, and D. Moher. Preferred reporting items for systematic reviews and meta-analysis: extension for scoping reviews (PRISMA-SCR). 2015; Available from: http://www.prisma-statement.org/Extensions/ InDevelopment.aspx.

28. Levac D, Colquhoun H, O'Brien KK. Scoping studies: advancing the methodology. Implement Sci. 2010;5(1):69.

29. Spencer, L., et al., Quality in qualitative evaluation: a framework for assessing research evidence. 2003, Government Chief Social Researcher's Office.

30. Galantino ML, et al. The effect of group aerobic exercise and t'ai chi on functional outcomes and quality of life for persons living with acquired immunodeficiency syndrome. J AlternComplement Med. 2005;11(6):1085-92.

31. McCain NL, et al. A randomized clinical trial of alternative stress management interventions in persons with HIV infection. J Consult Clin Psychol. 2008;76(3):431-41.

32. Koar WH. Correlates of meditation, depression, anxiety and T cell counts in HIV positive patients. United States -- California: University of Southern California; 1994. p. 61.

33. McCain NL, et al. \#97 tai chi training enhances well-being and alters cytokine levels in persons with HIV disease. Brain Behav Immun. 2005;19(4 Supplement):e50-1.

34. Robins JLW, et al. Research on psychoneuroimmunology: tai chi as a stress management approach for individuals with HIV disease. Appl Nurs Res. 2006;19(1):2-9

35. Nicholas PK, Webster A. A behavioral medicine intervention in persons with HIV. Clin Nurs Res. 1996;5(4):391-406.

36. Bonadies V. A yoga therapy program for AIDS-related pain and anxiety: implications for therapeutic recreation. Ther Recreat J. 2004;38(2):148-66.

37. Joseph B, Nair PM, Nanda A. Effects of naturopathy and yoga intervention on CD4 count of the individuals receiving antiretroviral therapy-report from a human immunodeficiency virus sanatorium, Pune. Int J Yoga. 2015;8(2):122-7.

38. Brazier A, Mulkins A, Verhoef M. Evaluating a yogic breathing and meditation intervention for individuals living with HIV/AIDS. Am J Health Promot. 2006:20(3):192-5.

39. Rao R, et al. P02.179. Effects of an integrated yoga program on mood, perceived stress, quality of life and immune measures in HIV patients: a pilot study. BMC Complement Altern Med. 2012;12(Suppl 1):1-1.

40. Menon JA, Glazebrook C. Randomized control trial to evaluate yoga-based peer support group for human immunodeficiency virus (HIV) positive Zambian adolescents. J AIDS HIV Res Vol. 2013;5(1):12-9.

41. Mawar N, et al. Sudarshan Kriya yoga improves quality of life in healthy people living with HIV (PLHIV): results from an open label randomized clinical trial. Indian J Med Res. 2015;141:90-9.

42. Agarwal RP, Kumar A, Lewis JE. A pilot feasibility and acceptability study of yoga/ meditation on the quality of life and markers of stress in persons living with HIV who also use crack cocaine. J Altern Complement Med. 2015;21(3):152-8.

43. Buti RL. The effects of meditation on global and factor scores on the BSI: A secondary analysis. United States -- Florida: Florida Atlantic University; 1998. p. 66.

44. Wood NA. Mindfulness-based cognitive therapy for the symptoms of depression in a community-based HIV/AIDS clinic: Outcomes and feasibility. United States -- Pennsylvania: Widener University, Institute for Graduate Clinical Psychology; 2009. p. 159.

45. Sibinga EMS, et al. Mindfulness-based stress reduction for urban youth. J Altern Complement Med. 2011;17(3):213-8.

46. Moskowitz JT, et al. A positive affect intervention for people experiencing health-related stress: development and non-randomized pilot test. J Health Psychol. 2012;17(5):676-92.

47. Price CJ, et al. Developing compassionate self-care skills in persons living with HIV: a pilot study to examine mindful awareness in body-oriented therapy feasibility and acceptability. Int J Ther Massage Bodywork. 2013;6(2):9-19.
48. O'Donovan A, Gibson S, Gill E. Compassion-focused therapy for people living with HIV: pilot of a mindfulness and compassion-based cognitive therapy group. HIV Med. 2015;16:63.

49. Robinson FP. Psycho-endocrine-immune response to mindfulness-based stress reduction in HIV-infected individuals. United States -- Illinois: Loyola University of Chicago; 2002. p. 200

50. Ampunsiriratana A, et al. A palliated-suffering model for HIV-infected patients: a combination of the foundations of mindfulness meditation and Watson's caring. Thai J Nurs Res. 2005;9(4):268-80.

51. Margolin A, et al. A preliminary study of spiritual self-schema (3-S+) therapy for reducing impulsivity in HIV positive drug users. J Clin Psychol. 2007;63(10):979-99.

52. Weinberger MC. The comparative efficacy of stress inoculation training and supportive therapy in the treatment of psychological distress in males with AIDS-related complex. United States -- California: California School of Professional Psychology - San Diego; 1988. p. 233.

53. Williams $A L$, et al. A randomized controlled trial of meditation and massage effects on quality of life in people with late-stage disease: a pilot study. J Palliat Med. 2005;8(5):939-52.

54. Bormann JE, et al. Effects of spiritual mantram repetition on HIV outcomes: a randomized controlled trial. J Behav Med. 2006;29(4):359-76.

55. Creswell JD. Mindfulness, stress, and immunity in HIV. Brain Behav Immun. 2009;23:S32

56. Seyedalinaghi $\mathrm{S}$, et al. Randomized controlled trial of mindfulness-based stress reduction delivered to human immunodeficiency virus-positive patients in Iran: effects on CD4 + T lymphocyte count and medical and psychological symptoms. Psychosom Med. 2012;74(6):620-7.

57. Duncan LG, et al. Mindfulness-based stress reduction for HIV treatment side effects: a randomized, wait-list controlled trial. J Pain Symptom Manag. 2012:43(2):161-71

58. Gayner B, et al. A randomized controlled trial of mindfulness-based stress reduction to manage affective symptoms and improve quality of life in gay men living with HIV. J Behav Med. 2012;35(3):272-85.

59. Mishra PH, Kumaraiah V, Chandra PS. Cognitive behavioural intervention in HIV-infected cases. NIMHANS J. 1998;16(1):37-41.

60. Safren SA, et al. Cognitive-behavioral therapy for HIV medication adherence and depression. Cogn Behav Pract. 2004;11(4):415-24.

61. Bommareddi $P$, Valsaraj BP. Jacobson's progressive muscle relaxation (JPMR) training to reduce anxiety and depression among people living with HIV. Nitte Univ J Health Sci. 2014;4(1):72-8.

62. Molassiotis A, et al. A pilot study of the effects of cognitive-behavioral group therapy and peer support/counseling in decreasing psychologic distress and improving quality of life in Chinese patients with symptomatic HIV disease. AIDS Patient Care STDs. 2002;16(2):83-96.

63. Antoni $\mathrm{MH}$, et al. Cognitive-behavioral stress management intervention buffers distress responses and immunologic changes following notification of HIV-1 seropositivity. J Consult Clin Psychol. 1991;59(6):906-15.

64. Kelly JA, et al. Outcome of cognitive-behavioral and support group brief therapies for depressed, HIV-infected persons. Am J Psychiatry. 1993;150(11):1679-86.

65. Mulder D, et al. Decreasing HIV-1 seroprevalence in young adults in a rural Ugandan cohort. BMJ. 1995;311(7009):833-6.

66. Fukunishi I, et al. Liaison psychiatry and HIV infection (II): application of relaxation in HIV positive patients. PsychiatryClin Neurosci. 1997;51(1):5-8.

67. Diego MA, et al. HIV adolescents show improved immune function following massage therapy. Int J Neurosci. 2001;106(1-2):35-45.

68. Langenfeld MC. The effects of hypnosis on pain-control with people living with HIV-AIDS. United States -- California: California School of Professional Psychology - Fresno; 1999. p. 89.

69. Rucklidge JJ, Saunders D. The efficacy of hypnosis in the treatment of pruritus in people with HIV/AIDS: a time-series analysis. Int J Clin Exp Hypn. 2002;50(2):149-69.

70. Biswas UN. Positive thought induction for arresting disease progression: a hypnotherapeutic application in HIV/AIDS. Psychol Stud. 2011;56(2):192-205.

71. Dorfman D, et al. Hypnosis for treatment of HIV neuropathic pain: a preliminary report. Pain Med. 2013;14(7):1048-56.

72. Vieille RC Jr. The use of hypnosis, self-hypnosis, mental imagery, and naturalistic trance to enhance immunity and health in gay men with HIV: A contextualistic perspective. United States -- California: California School of Professional Psychology - Fresno; 1997. p. 310.

73. Gill ST. An Ericksonian hypnosis intervention on psychological distress and immune functioning in HIV-1 seropositive patients. United States -- Florida: University of Miami; 2000. p. 83. 
74. Shrier LS. The effect of hypnosis on the immune systems of AIDS and HIVinfected patients. United States - Pennsylvania: Temple University; 1992. p. 149.

75. Kacker P. Hypnotherapy as an effective modulation for enhancing subjective vitality of people living with HIV/AIDS and dermatitis. Int J. 2015;3(4):928-35.

76. Benita IC. Effects of guided imagery paired with a relaxation technique on people with HIV-related illnesses. United States -- Connecticut: Southern Connecticut State University; 1989. p. 45.

77. Donaldson WW. A clinical study of visualization on depressed white blood cell count in medical patients. Appl Psychophysiol Biofeedback. 2000;25(2):117-28.

78. Hickey KA. The effect of caring massage and guided relaxation imagery on well-being of medical patients in a hospital setting. United States -California: California Institute of Integral Studies; 1993. p. 144.

79. Sussman PR. A comparison of palliative stress reduction versus problem-solving training in the treatment of HIV seropositive males. United States -- California: California School of Professional Psychology - San Diego; 1990. p. 197.

80. Eller LS. Guided imagery: a nursing intervention for symptoms related to infection with human immunodeficiency virus [dissertation]; 1994.

81. Brown $\mathrm{J}$, et al. Computerized stress management training for HIV+ women: a pilot intervention study. AIDS Care. 2011;23(12):1525-32.

82. Kermani KS. Stress, emotions, autogenic training and AIDS: a holistic approach to the management of HIV-infected individuals. Holistic Med. 1987;2(4):203-15.

83. Tarakeshwar N, Pearce MJ, Sikkema KJ. Development and implementation of a spiritual coping group intervention for adults living with HIV/AIDS: a pilot study. Ment Health Relig Cult. 2005;8(3):179-90.

84. Schummer GJ, Noh SM, Mendoza JJ. The effect of neurofeedback and cranial electrotherapy on immune function within a group of HIV+ subjects: a controlled study. J Neurother. 2013;17(3):151-61.

85. Lundy ST. Toward an integral aids therapy: psycho-social factors and stress management in living with aids (Biofeedback). United States -- California: California Institute of Integral Studies; 1986. p. 177.

86. Kearl AM. Stress reduction, immune response, and human immunodeficiency virus: Treatment combining biofeedback, music, relaxation, and guided imagery. United States -- California: University of the Pacific; 1991. p. 178.

87. Chandler CK, Sanders P. Biofeedback therapy with an HIV-positive subject. TCA J. 1994;22(1):40-7.

88. McCain $\mathrm{NL}$, et al. The influence of stress management training in HIV disease. Nurs Res. 1996;45(4):246-53.

89. Trafton JA, et al. Outcomes associated with a cognitive-behavioral chronic pain management program implemented in three public HIV primary care clinics. J. Behav. Health Serv. Res. 2012;39(2):158-73.

90. Jones $\mathrm{DL}$, et al. Translation of a comprehensive health behavior intervention for women living with HIV: the SMART/EST Women's program. Transl Behav Med. 2013;3(4):416-25.

91. Auerbach JE, Oleson TD, Solomon GF. A behavioral medicine intervention as an adjunctive treatment for HIV-related illness. Psychol Health. 1992;6(4):325-34.

92. Taylor DN. Effects of a behavioral stress-management program on anxiety, mood, self-esteem, and T-cell count in HIV positive men. Psychol Rep. 1995; 76(2):451-7.

93. Lutgendorf SK, et al. Cognitive-behavioral stress management decreases dysphoric mood and herpes simplex virus-type 2 antibody titers in symptomatic HIV-seropositive gay men. J Consult Clin Psychol. 1997;65(1):31-43.

94. Gifford $A L$, et al. Pilot randomized trial of education to improve selfmanagement skills of men with symptomatic HIV/AIDS. J Acquir Immune Defic Syndr Hum Retrovirol. 1998;18(2):136-44.

95. Stout-Shaffer SR. Effects of relaxation training on physiological and psychological measures of distress and quality of life in HIV-seropositive subjects [dissertation]; 1999.

96. Birk TJ, et al. The effects of massage therapy alone and in combination with other complementary therapies on immune system measures and quality of life in human immunodeficiency virus. J Altern Complement Med. 2000;6(5):405-14.

97. Inouye J, Flannelly L, Flannelly KJ. The effectiveness of self-management training for individuals with HIV/AIDS. [Republished in J Assoc Nurses AIDS Care. 2001 Sep-Oct;12(5):71-82; PMID: 11565240]. J Assoc Nurses AIDS Care. 2001;12(2):73-84.

98. Cruess $\mathrm{S}$, et al. Changes in mood and depressive symptoms and related change processes during cognitive-behavioral stress management in HIVinfected men. Cogn Ther Res. 2002;26(3):373-92.

99. McCain NL, et al. Effects of stress management on PNI-based outcomes in persons with HIV disease. Res Nurs Health. 2003;26(2):102-17.
100. Antoni MH, et al. Randomized clinical trial of cognitive behavioral stress management on human immunodeficiency virus viral load in gay men treated with highly active antiretroviral therapy. Psychosom Med. 2006;68(1): 143-51.

101. Chang $\mathrm{BH}$, et al. The combined effect of relaxation response and acupuncture on quality of life in patients with HIV: a pilot study. J Altern Complement Med. 2007;13(8):807-15.

102. Antoni $\mathrm{MH}$, et al. Stress management effects on perceived stress and cervical neoplasia in low-income HIV-infected women. J Psychosom Res. 2008;65(4):389-401.

103. Jones DL, et al. Self-efficacy and distress in women with AIDS: the SMART/ EST women's project. AIDS Care. 2010;22(12):1499-508.

104. Chang BH, Sommers E. Acupuncture and the relaxation response for treating gastrointestinal symptoms in HIV patients on highly active antiretroviral therapy. Acupunct Med. 2011:29(3):180-7.

105. Soroudi N, et al. CBT for medication adherence and depression (CBT-AD) in HIV-infected patients receiving methadone maintenance therapy. Cogn Behav Pract. 2008;15(1):93-106.

106. Fawzy FI, Namir S, Wolcott DL. Structured group intervention model for AIDS patients. Psychiatr Med. 1989;7(2):35-45.

107. Coates TJ, et al. Stress reduction training changed number of sexual partners but not immune function in men with HIV. Am J Public Health. 1989;79(7):885-7.

108. Perry $\mathrm{S}$, et al. Effectiveness of psychoeducational interventions in reducing emotional distress after human immunodeficiency virus antibody testing. Arch Gen Psychiatry. 1991;48(2):143-7.

109. Messinis, L., The effects of an individualised cognitive-behavioral and electromyographic feedback intervention on HIV-seropositive patients. (Doctoral dissertation). 1997.

110. Chesney MA, et al. Coping effectiveness training for men living with HIV: results from a randomized clinical trial testing a group-based intervention. Psychosom Med. 2003;65(6):1038-46.

111. Weiss JJ, et al. Effects of a supportive-expressive group intervention on long-term psychosocial adjustment in HIV-infected gay men. Psychother Psychosom. 2003;72(3):132-40.

112. Chan I, et al. Cognitive-behavioral group program for Chinese heterosexual HIV-infected men in Hong Kong. Patient Educ Couns. 2005;56(1):78-84.

113. Mondy K, et al. Hatha/Ashtanga yoga intervention modestly improves cardiovascular disease (CVD) risk parameters in dyslipidaemic HIV+ subjects with central adiposity. Antivir Ther. 2007;12(6):L47-8.

114. Menon JA, et al. Evaluation of a yoga-based support group for HIV positive, Zambian adolescents. Psychol Health. 2008;23:183-4.

115. Sibinga EMS, et al. Mindfulness-based stress reduction for HIV-infected youth: a pilot study. Explore. 2008;4(1):36-7.

116. Kerrigan D, et al. Perceptions, experiences, and shifts in perspective occurring among urban youth participating in a mindfulness-based stress reduction program. Complement Ther Clin Pract. 2011;17(2):96-101.

117. Beitel $M$, et al. Reflections by inner-city drug users on a Buddhist-based spirituality-focused therapy: a qualitative study. Am J Orthopsychiatry. 2007; 77(1):1-9.

118. Bormann JE, et al. Effects of faith/assurance on cortisol levels are enhanced by a spiritual mantram intervention in adults with HIV: a randomized trial. J Psychosom Res. 2009;66(2):161-71.

119. Creswell JD. Biobehavioral effects of mindfulness-based stress reduction in HIV. United States -- California: University of California, Los Angeles; 2007. p. 73-n/a.

120. Jam S, et al. The effects of mindfulness-based stress reduction (MBSR) program in Iranian HIV/AIDS patients: a pilot study. Acta Med Iran. 2010; 48(2):101-6

121. Andrade AS, et al. Pharmacokinetic and metabolic effects of American ginseng (Panax quinquefolius) in healthy volunteers receiving the HIV protease inhibitor indinavir. BMC Complement Altern Med. 2008;8:50.

122. Prachi, et al. Cognitive behaviour therapy in HIV-infection: A case study. Journal of Personality and Clinical Studies. 1998;14(1-2):41-6.

123. Helder LM. The effects of a cognitive/behavioral stress management program on psychological distress and the immune system in HIV-1 seropositive and seronegative gay men. United States -- Florida: University of Miami; 1992. p. 164.

124. Esterling BA. Relaxation and exercise intervention as a means of modulating antibody to Epstein-Barr and human herpesvirus type- 6 in an asymptomatic HIV-1 seropositive and seronegative cohort; 1991. 
125. Baggett HL. Coping with HIV-1 serostatus notification. United States Florida: University of Miami; 1991. p. 107.

126. Antoni $\mathrm{MH}$, et al. Stress and immunity in individuals at risk for AIDS. Stress Med. 1991;7(1):35-44.

127. Baggett $H L$, et al. The effects of frequency of relaxation practice on immune markers IN AN HIV-1 high-risk group. Psychosom Med. 1990;52(2):243.

128. Langenfeld MC, Cipani E, Borckardt JJ. Hypnosis for the control of HIV/AIDSrelated pain. Int J Clin Exp Hypn. 2002;50(2):170-88.

129. Dorfman D, et al. (332) Hypnosis for treatment of HIV neuropathic pain. J Pain. 2008;9(4 Supplement 2):59.

130. Donaldson WW. The effects of visualization on depressed white blood cell count in medical patients. United States -- Ohio: The Union for Experimenting Colleges and Universities; 1988. p. 110.

131. Eller LS. Effects of two cognitive-behavioral interventions on immunity and symptoms in persons with HIV. Ann Behav Med. 1995;17(4):339-48.

132. Eller LS. Effects of cognitive-behavioral interventions on quality of life in persons with HIV. Int J Nurs Stud. 1999;36(3):223-33.

133. Brown $J \mathrm{~L}$, et al. The development of a computer-administered cognitivebehavioral intervention to promote stress management among HIV+ women. J Cogn Psychother. 2010;24(4):265-80.

134. Schummer G, Noh S. The effect of neurofeedback and cranial electrotherapy on immune function within a group of HIV+subjects: a randomized controlled study. J Neurother. 2011;15(4):427-8.

135. Cucciare MA, Sorrell JT, Trafton JA. Predicting response to cognitivebehavioral therapy in a sample of HIV-positive patients with chronic pain. J Behav Med. 2009;32(4):340-8.

136. Weiss SM, et al. Translating an evidence-based behavioral intervention for women living with HIV into clinical practice: the SMART/EST Women's program. Int J Behav Med. 2015;22(3):415-24.

137. Lutgendorf SK, et al. Changes in cognitive coping skills and social support during cognitive behavioral stress management intervention and distress outcomes in symptomatic human immunodeficiency virus (HIV)-seropositive gay men. Psychosom Med. 1998;60(2):204-14.

138. Antoni $\mathrm{MH}$, et al. CBSM intervention effects on mood and immunity in HIV+ gay men: the role of relaxation training, coping skills and social support. Psychosom Med. 1997;59(1):81-2.

139. Lutgendorf SK. Cognitive-behavioral stress management in a symptomatic HIV-1 seropositive population: Effects on mood, coping, immune and neuroendocrine factors. United States -- Florida: University of Miami; 1994. p. 286.

140. Lutgendorf SK, et al. Can relaxation modulate neuroendocrine functioning even in progressed HIV disease. Psychosom Med. 1995;57(1):80.

141. Gifford AL, Sengupta S. Self-management health education for chronic HIV infection. AIDS Care. 1999;11(1):115-30.

142. Cruess DG, et al. Reductions in salivary cortisol and anxious mood during three different forms of relaxation training in symptomatic, HIV+ men. Psychosom Med. 1999;61(1):127.

143. Antoni MH, et al. Cognitive-behavioral stress management intervention effects on anxiety, 24-hr urinary norepinephrine output, and T-cytotoxic/ suppressor cells over time among symptomatic HIV-infected gay men. J Consult. Clin Psychol. 2000;68(1):31-45.

144. Cruess DG, et al. Reductions in salivary cortisol are associated with mood improvement during relaxation training among HIV-seropositive men. J Behav Med. 2000;23(2):107-22.

145. Cruess DG, et al. Cognitive-behavioral stress management increases free testosterone and decreases psychological distress in HIV-seropositive men. Health Psychol. 2000;19(1):12-20.

146. Cruess S, et al. Reductions in herpes simplex virus type 2 antibody titers after cognitive behavioral stress management and relationships with neuroendocrine function, relaxation skills, and social support in HIV-positive men. Psychosom Med. 2000;62(6):828-37.

147. Antoni $\mathrm{MH}$, et al. Cognitive-behavioral stress management reduces distress and 24-hour urinary free cortisol output among symptomatic HIV-infected gay men. Ann Behav Med. 2000;22(1):29-37.

148. Cruess DG. Changes in depressive symptoms, distress, and hpa and HPA and HPG axis hormones during cognitive-behavioral stress management in symptomatic HIV-positive gay men (Doctoral dissertation); 1998.

149. Carrico AW, et al. Cognitive-behavioural stress management with HIVpositive homosexual men: mechanisms of sustained reductions in depressive symptoms. Chronic IIIn. 2005;1(3):207-15.

150. Antoni $\mathrm{MH}$, et al. Stress management and immune system reconstitution in symptomatic HIV-infected gay men over time: effects on transitional naive T cells (CD4(+)CD45RA(+)CD29(+)). Am J Psychiatry. 2002;159(1):143-5.

151. Carrico AW, et al. Reductions in depressed mood and denial coping during cognitive behavioral stress management with HIV-positive gay men treated with HAART. Ann Behav Med. 2006;31(2):155-64.

152. Chang $\mathrm{BH}$, et al. Relaxation response with acupuncture trial in patients with HIV: feasibility and participant experiences. J Altern Complement Med. 2007; 13(7):719-24.

153. Danielson AL. Emotional status and social support relations to immune status in women co-infected with HIV and HPV: Preliminary evidence for beneficial psychosocial effects of a CBSM intervention tailored for this population (Doctoral dissertation); 2002.

154. Segal-Isaacson CJ, et al. Improving dietary habits in disadvantaged women with HIV/AIDS: the SMART/EST women's project. AIDS Behav. 2006;10(6):659-70.

155. Lechner SC, et al. Cognitive-behavioral interventions improve quality of life in women with AIDS. J Psychosom Res. 2003;54(3):253-61.

156. Laperriere A, et al. Decreased depression up to one year following CBSM+ intervention in depressed women with AIDS: the Smart/EST Women's project. J Health Psychol. 2005;10(2):223-31.

157. Jones $\mathrm{DL}$, et al. Efficacy of a group medication adherence intervention among HIV positive women: the SMART/EST Women's project. AIDS Behav. 2007;11(1):79-86.

158. Jones $\mathrm{DL}$, et al. Influencing medication adherence among women with AIDS. AIDS Care. 2003;15(4):463-74

159. Jones $D$, et al. The effect of relaxation interventions on cortisol levels in HIVseropositive women. J Int Assoc Provid AIDS Care. 2014;13(4):318-23.

160. Jones $D L$, et al. A brief sexual barrier intervention for women living with AIDS: acceptability, use, and ethnicity. J Urban Health. 2001;78(4): 593-604.

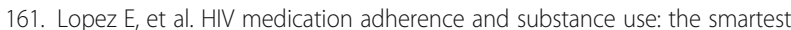
Women's project. Am J Infect Dis. 2007;3(4):240-7.

162. Messinis L, Antoniadis G, Spiropouloy P. Cognitive-behavioral therapy and EMG biofeedback as an adjunctive treatment method for asymptomatic AIDS patients. ENCEPHALOS-ATHENS. 2002;39(2):103-24.

163. Chesney M, Folkman S, Chambers D. Coping effectiveness training for men living with HIV: preliminary findings. Int J STD AIDS. 1996;7(Suppl 2):75-82.

164. Riley KE, Kalichman S. Mindfulness-based stress reduction for people living with HIV/AIDS: preliminary review of intervention trial methodologies and findings. Health Psychol Rev. 2015;9(2):224-43.

165. Chan RR, Larson JL. Meditation interventions for chronic disease populations: a systematic review. J Holist Nurs. 2015;33(4):351-65.

166. Gotink RA, et al. Standardised mindfulness-based interventions in healthcare: an overview of systematic reviews and meta-analyses of RCTs. PLoS One. 2015;10(4):e0124344.

167. Hall DL, et al. Telephone-administered versus live group cognitive behavioral stress management for adults with CFS. J Psychosom Res. 2017;93:41-7.

168. Russell DC, et al. Effects of cognitive behavioral stress management on negative mood and cardiac autonomic activity in ICD recipients. Pacing Clin Electrophysiol. 2015;38(8):951-65.

169. Stagl JM, et al. Long-term psychological benefits of cognitive-behavioral stress management for women with breast cancer: 11-year follow-up of a randomized controlled trial. Cancer. 2015;121(11):1873-81.

170. Manzoni GM, et al. Relaxation training for anxiety: a ten-years systematic review with meta-analysis. BMC Psychiatry. 2008:8:41.

171. Klainin-Yobas $P$, et al. Effects of relaxation interventions on depression and anxiety among older adults: a systematic review. Aging Ment Health. 2015; 19(12):1043-55.

172. Adachi T, et al. A meta-analysis of hypnosis for chronic pain problems: a comparison between hypnosis, standard care, and other psychological interventions. Int J Clin Exp Hypn. 2014;62(1):1-28.

173. Zech $N$, et al. Efficacy, acceptability and safety of guided imagery/hypnosis in fibromyalgia - a systematic review and meta-analysis of randomized controlled trials. Eur J Pain. 2017;21(2):217-27.

174. Stetter F, Kupper S. Autogenic training: a meta-analysis of clinical outcome studies. Appl Psychophysiol Biofeedback. 2002;27(1):45-98.

175. Luctkar-Flude M, Groll D. A systematic review of the safety and effect of neurofeedback on fatigue and cognition. Integr Cancer Ther. 2015;14(4):318-40.

176. Chu P, et al. The effectiveness of yoga in modifying risk factors for cardiovascular disease and metabolic syndrome: a systematic review and meta-analysis of randomized controlled trials. Eur J Prev Cardiol. 2016;23(3):291-307. 
177. Pascoe MC, Bauer IE. A systematic review of randomised control trials on the effects of yoga on stress measures and mood. J Psychiatr Res. 2015;68:270-82.

178. Chen YW, et al. The effect of tai chi on four chronic conditions-cancer, osteoarthritis, heart failure and chronic obstructive pulmonary disease: a systematic review and meta-analyses. Br J Sports Med. 2016;50(7):397-407.

179. Liu X, et al. A systematic review and meta-analysis of the effects of qigong and tai chi for depressive symptoms. Complement Ther Med. 2015;23(4):516-34.

180. Jahnke $\mathrm{R}$, et al. A comprehensive review of health benefits of qigong and tai chi. A J Health Promot. 2010;24(6):e1-e25.

181. Morgan N, et al. The effects of mind-body therapies on the immune system: meta-analysis. PLoS One. 2014;9(7):e100903.

182. Gupta SK. Intention-to-treat concept: a review. Perspect Clin Res. 2011;2(3):109-12.

183. Schulz KF, Altman DG, Moher D. CONSORT 2010 statement: updated guidelines for reporting parallel group randomised trials. Bmj. 2010;340:c332.

Ready to submit your research? Choose BMC and benefit from:

- fast, convenient online submission

- thorough peer review by experienced researchers in your field

- rapid publication on acceptance

- support for research data, including large and complex data types

- gold Open Access which fosters wider collaboration and increased citations

- maximum visibility for your research: over $100 \mathrm{M}$ website views per year

At BMC, research is always in progress.

Learn more biomedcentral.com/submissions 